\title{
Antiplatelet regimens for Asian patients with ischemic stroke or transient ischemic attack: a systematic review and network meta- analysis
}

\author{
Seung Jin Jung ${ }^{1 \#}$, Sung-Ryul Shim ${ }^{2 \#}$, Bum Joon Kim ${ }^{3}$, Jin-Man Jung ${ }^{4,5} \wedge$ \\ ${ }^{1}$ Department of Family Medicine, Gimpo Woori Hospital, Gimpo, Republic of Korea; ${ }^{2}$ Department of Preventive Medicine, Korea University \\ College of Medicine, Seoul, Republic of Korea; ${ }^{3}$ Department of Neurology, Asan Medical Center, Seoul, Republic of Korea; ${ }^{4}$ Department of \\ Neurology, Korea University Ansan Hospital, Ansan, Republic of Korea; ${ }^{5}$ Korea University Zebrafish Translational Medical Research Center, Ansan, \\ Republic of Korea \\ Contributions: (I) Conception and design: JM Jung; (II) Administrative support: JM Jung; (III) Provision of study materials or patients: SJ Jung, JM \\ Jung; (IV) Collection and assembly of data: SJ Jung, BJ Kim, JM Jung; (V) Data analysis and interpretation: SR Shim; (VI) Manuscript writing: All \\ authors; (VII) Final approval of manuscript: All authors \\ \#These authors contributed equally to this work as the first author. \\ Correspondence to: Jin-Man Jung, MD, PhD. Department of Neurology, Korea University Ansan Hospital, 123, Jeokgeum-ro, Danwon-Gu, Ansan-Si, \\ Gyeonggi-Do 15355, South Korea. Email: dr.jinmanjung@gmail.com.
}

Background: The optimal antiplatelet treatment for the secondary prevention of non-cardioembolic stroke or transient ischemic attack (TIA) remains uncertain in Asians.

Methods: We searched for eligible randomized control trials in Medline, Embase, and the Cochrane Library. A Bayesian network meta-analysis (NMA) was performed to assess the efficacy and safety of antiplatelet regimens with placebo as the control. Each therapy was compared using relative risk ratios (RR) and $95 \%$ credible intervals $(\mathrm{CrI})$, and ranked according to the value of the surface under the cumulative ranking curve.

Results: A total of 84,103 patients from 32 studies were included: patients in used aspirin $(\mathrm{n}=26,834)$; cilostazol ( $n=3,303)$; clopidogrel $(n=12,406)$; prasugrel $(n=1,885)$; sarpogrelate $(n=752)$; ticagrelor $(n=1,933)$; ticlopidine $(n=1,644)$; triflusal $(n=391)$; aspirin plus cilostazol $(n=1,120)$, aspirin plus clopidogrel $(n=4,623)$; aspirin plus dipyridamole $(n=10,853)$; aspirin plus ticagrelor $(n=5,859)$; aspirin plus ticlopidine $(n=132)$. Patients who used aspirin plus clopidogrel and cilostazol had a lower risk of recurrent stroke than those who used placebo. Patients administered with aspirin plus ticagrelor, aspirin plus clopidogrel, and cilostazol had a lower risk of composite vascular events than those administered placebo. Patients administered aspirin plus ticagrelor had a higher risk of major bleeding than those administered placebo. Clustered three-dimensional rank plots of recurrent stroke, major bleeding, and composite vascular events demonstrated that cilostazol had higher values of the surface under the cumulative ranking curve than other treatments.

Conclusions: Of the antiplatelet regimens, cilostazol showed the best net clinical benefits than other antiplatelet regimens in Asians with non-cardioembolic stroke or TIA.

Keywords: Antiplatelet agents; network meta-analysis (NMA); secondary prevention; Asian; stroke

Submitted Dec 12, 2020. Accepted for publication Mar 10, 2021.

doi: 10.21037/atm-20-7951

View this article at: http://dx.doi.org/10.21037/atm-20-7951

\footnotetext{
$\wedge$ ORCID: 0000-0003-0557-6431.
} 


\section{Introduction}

Antiplatelet treatment is the main strategy for the secondary prevention of vascular events in patients with non-cardioembolic stroke or transient ischemic attack (TIA) $(1,2)$. Previous guidelines have recommended aspirin, clopidogrel, aspirin plus clopidogrel, and aspirin plus dipyridamole for secondary prevention (3). However, these are mostly based on clinical trial results from Western populations.

Several characteristics of ischemic stroke (IS) in Asian populations differ from those in Western populations. The stroke mortality and incidence rates are higher (4), and IS due to intracranial atherosclerotic stenosis and smallvessel occlusion is more frequent in Asian than in Western populations (5). The high prevalence of small-vessel disease is associated with an increased risk of cerebral hemorrhage. The risk of bleeding, including gastrointestinal bleeding, is also higher in Asians than in Westerners (6). This may be partially attributed to the high prevalence of Helicobacter pylori infection and genetic differences (7). The metabolisms of specific antiplatelet agents are also affected by genetic variance, which may also affect the efficacy and safety in patients with specific phenotypes more frequently observed in Asians.

Based on these findings, antiplatelet agents with reduced risks of bleeding may be potentially beneficial in Asian populations. However, no meta-analysis on the optimal antiplatelet agent for Asians has been conducted. Here, we performed a systematic review and network meta-analysis (NMA) to assess the comparative efficacy and safety of antiplatelet regimens for secondary prevention after noncardioembolic IS or TIA in Asians.

We describe the contents in accordance with the PRISMA NMA reporting checklist (available at http:// dx.doi.org/10.21037/atm-20-7951).

\section{Methods}

This systematic review follows the principles in the Preferred Reporting Items for Systematic Reviews and Meta-Analyses statement (8).

\section{Search strategy}

We used multiple comprehensive databases (Medline, Embase, and Cochrane Library) to identify relevant studies from inception to May 26, 2020. The search terms included "ischemic stroke", "transient ischemic attack", "secondary prevention", and "antiplatelet agents". No restrictions on language were set. The detailed search strategies are presented in Table S1. The searched articles were reviewed in two steps by two independent reviewers (SJ Jung and JM Jung). An initial search was performed using the titles and abstracts, after which a further full-text review was performed. A manual search using additional sources, such as reference lists, was also performed. We contacted the relevant authors to obtain more information, if necessary.

\section{Study selection}

Studies were included if they were randomized and headto-head trials that compared the efficacy and safety of antiplatelet regimens for the secondary prevention of noncardioembolic stroke and/or TIA. Studies were excluded if they (I) investigated diseases other than IS or TIA, such as coronary artery disease or peripheral vascular disease, (II) compared anti-coagulant drugs or aspirin doses beyond the range of 50-330 mg, and (III) included only non-Asian populations. However, if we could find and extract the Asian population results of the global trials conducted on two or more continents, we included the results from the subgroup analysis. For international trials without a subgroup analysis based on ethnicity, only trials with more than $30 \%$ of Asian patients were included. For the trials with an extended follow-up, only those with follow-up periods according to the original study design were included. Different opinions of the two independent reviewers were resolved through consensus.

\section{Data extraction and quality assessment}

Two independent reviewers extracted the data using a predefined data extraction template. The data from the eligible trials included the following: basal characteristics (ethnicity, sex, age, stroke subtype, and underlying diseases such as hypertension and diabetes), detailed characteristics of the study (design, type of intervention drug, dosage, sample size, onset-to-treatment time, duration of total treatment, combination treatment, and follow-up), and indicators of the treatment effect such as the frequencies of recurrent strokes, recurrent IS, composite vascular events (stroke, myocardial infarction, and vascular death), all forms of bleeding, and major bleeding. The primary efficacy outcome was a recurrent stroke, and the primary safety outcome was major bleeding. The secondary efficacy outcomes were recurrent IS and composite vascular events, and the secondary safety outcome was all bleeding. For the 
trials that did not report on the outcomes of interest, the value obtained by adding or subtracting the values from other resources, including relevant articles and previous meta-analyses, was used. For multi-arm trials involving antiplatelet agents and other drugs, we extracted two or more interesting comparison arms and ignored the others.

The risk of bias for each study was assessed using the Cochrane risk of bias assessment tool (9). The risks of bias for the domains were categorized as low risk, unclear risk, or high risk. The risk of bias was assessed by two independent reviewers, and any disagreements were resolved through a discussion.

\section{Statistical analysis}

We performed a Bayesian NMA, using the $\mathrm{R}$ version 3.6 "gemtc" package. The analysis pooled the relative risk ratios (RR) and $95 \%$ credible intervals (CrI) using the number of patients experiencing index events and the total number of patients in an intention-to-treat population. A two-sided $\mathrm{P}$ value of $<0.05$ was considered statistically significant. Placebo or aspirin was used as a common comparator. For the inconsistency test, we performed node-splitting assessments to determine the association between the direct and indirect evidence. If no statistical significance was observed, the evidence was presumed to be consistent for the direct and indirect comparisons. Publication bias was examined using funnel plots. The antiplatelet regimens were ranked based on the surface under the cumulative ranking curve (SUCRA) probabilities and the rankograms. The SUCRA is expressed as a percentage ranging from 0-100\%. A higher SUCRA value indicates a higher ranking of a specific treatment; a top rank or one of the top ranks. Finally, the net clinical benefit (NCB) was determined using three-dimensional clustered rank plots and SUCRA ranking probabilities and used to assess the primary efficacy, safety, and composite vascular outcomes.

Subgroup analyses based on the symptom onset-totreatment duration of the antiplatelet agents $(\leq 72$ vs. $>72$ hours) were used to discriminate against the effect according to the period with a higher ischemic burden than bleeding risk.

\section{Results}

\section{Literature search results}

According to our search strategies, 1,571 relevant publications (460 from Medline, 768 from Embase, and
343 from Cochrane Library) were initially identified, and 49 additional records were found from other sources. Of them, 32 eligible articles were finally included in this NMA (Figure 1). The symptom onset-to-treatment duration of fourteen trials (10-23) was within 72 hours, and that of eighteen trials (24-41) was after 72 hours.

\section{Study characteristics and network formation}

The 32 included trials tested 13 antiplatelet regimens, including aspirin, cilostazol, clopidogrel, prasugrel, sarpogrelate, ticagrelor, ticlopidine, triflusal, aspirin plus cilostazol, aspirin plus clopidogrel, aspirin plus dipyridamole, aspirin plus ticagrelor, and aspirin plus ticlopidine. Thirty trials included only Asians. The proportions of the Asian population in the PRoFESS (29) and THALES (23) global trials were $32 \%$ and $42 \%$, respectively. Detailed characteristics of the included trials are presented in Table S2. Of the 32 eligible trials, 31 had two intervention arms. One trial (39) compared three intervention arms, but it compared different doses of clopidogrel plus aspirin (aspirin $100 \mathrm{mg}$ plus clopidogrel $50 \mathrm{mg}$ once daily $v s$. aspirin $100 \mathrm{mg}$ plus clopidogrel 75 $\mathrm{mg}$ once daily) with that of aspirin. Two different doses of clopidogrel were grouped and analyzed as two treatment arms. The mean age of the patients was 64 years. The mean incidence of hypertension and diabetes at baseline were $62 \%$ and $28 \%$, respectively. The mean duration of followup was 19 months, and the duration of follow-up was one month $(10,16,19,23)$ or less $(12,17)$, in six trials. Figure 2 shows the network plots of antiplatelet regimens.

\section{Risk of bias}

Of the 32 eligible trials, some showed indicators of a high or unclear risk of bias: random sequence generation $(n=5$, $15.6 \%)$, allocation concealment $(n=4,12.5 \%)$, blinding of participants and personnel $(n=13,40.6 \%)$, blinding of outcome assessment $(n=10,31.2 \%)$, and other bias $(n=2$, $6.2 \%$ ). The detailed characteristics of the risk of bias in the included trials are provided in Figures S1 and S2.

\section{Outcomes of interest}

\section{Recurrent stroke}

Thirty-one trials, with a sample size of 84,113 , reported recurrent stroke events. Figure 3 shows the results of the NMA. Aspirin plus clopidogrel ( $\mathrm{RR}=0.53$, 95\% CrI: $0.27-$ 


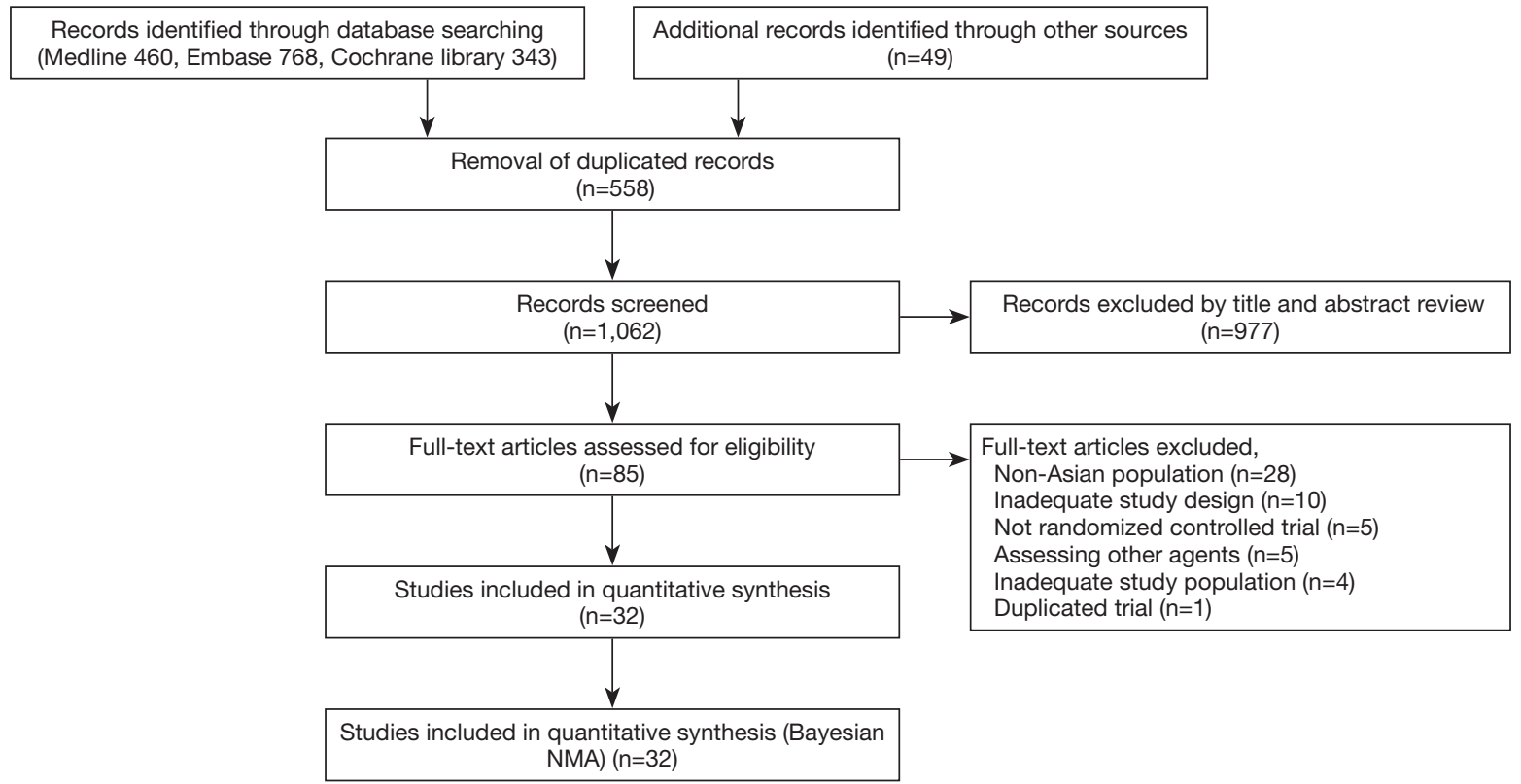

Figure 1 PRISMA flow chart.

0.83 ) and cilostazol ( $\mathrm{RR}=0.58,95 \% \mathrm{CrI}$ : 0.36-0.91) were associated with significantly lower risks of recurrent stroke than placebo. They were also associated with a lower risk of recurrent stroke than aspirin [RR, 95\% CrI; 0.57 (0.39-0.75) and 0.64 (0.46-0.88), respectively; Table S3]. Other antiplatelet regimens were not significantly more effective than placebo in preventing recurrent stroke. Based on the SUCRA values and the rankogram, aspirin plus clopidogrel ranked first, followed by cilostazol (Table S4 and Figure S3).

\section{Recurrent IS}

Thirty-two trials reported recurrent IS with a sample size of 85,982. As shown in Figure 3, aspirin plus clopidogrel ( $\mathrm{RR}=0.41,95 \% \mathrm{CrI}: 0.20-0.67)$, aspirin plus ticagrelor (RR $=0.48,95 \%$ CrI: 0.21-0.84), and cilostazol $(\mathrm{RR}=0.56,95 \%$ CrI: $0.34-0.90)$ were associated with significantly lower risks of recurrent IS than placebo. The other antiplatelet regimens were not more effective than placebo. Aspirin plus clopidogrel $(\mathrm{RR}=0.54,95 \% \mathrm{CrI}$ : $0.35-0.72)$ and aspirin plus ticagrelor $(\mathrm{RR}=0.65,95 \% \mathrm{CrI}$ : $0.34-0.94)$ were also associated with a lower risk of recurrent IS than aspirin (Table S3). Aspirin plus ticagrelor ranked first, followed by aspirin plus clopidogrel, aspirin plus cilostazol, and cilostazol (Table S4 and Figure S3). The efficacy of aspirin plus cilostazol was not significant although it ranked third
$(\mathrm{RR}=0.54,95 \%$ CrI: $0.24-1.15)$.

\section{Composite vascular events}

Twenty-one trials, with a sample size of 74,728, reported composite vascular events. Aspirin plus ticagrelor (RR $=0.38,95 \%$ CrI: 0.19-0.77), aspirin plus clopidogrel $(\mathrm{RR}$ $=0.56,95 \%$ CrI: $0.37-0.74)$, and cilostazol $(\mathrm{RR}=0.61,95 \%$ CrI: $0.45-0.80)$ were associated with significantly lower risks of composite vascular events than placebo (Figure 3); they were also associated with a lower risk of composite vascular events than aspirin [RR, 95\% CrI: 0.44 (0.23-0.87) for aspirin plus ticagrelor, $0.65(0.49-0.79)$ for aspirin plus clopidogrel, and $0.71(0.57-0.88)$ for cilostazol]. The other antiplatelet regimens were not more effective than placebo in preventing composite vascular events; aspirin plus ticagrelor ranked first, aspirin plus clopidogrel second, and cilostazol ranked third (Table S4 and Figure S3).

\section{Major bleeding}

Twenty-eight trials, with a sample size of 81,087 , reported major bleeding. Most antiplatelet regimens, except aspirin plus ticagrelor $(\mathrm{RR}=3.74,95 \% \mathrm{CrI}: 1.24-10.17)$, were not associated with a higher risk of major bleeding than placebo (Figure 3). Compared with aspirin, aspirin plus ticagrelor ( $\mathrm{RR}=2.82,95 \% \mathrm{CrI}: 1.24-6.04)$ was associated with a higher risk of major bleeding, whereas cilostazol 
A

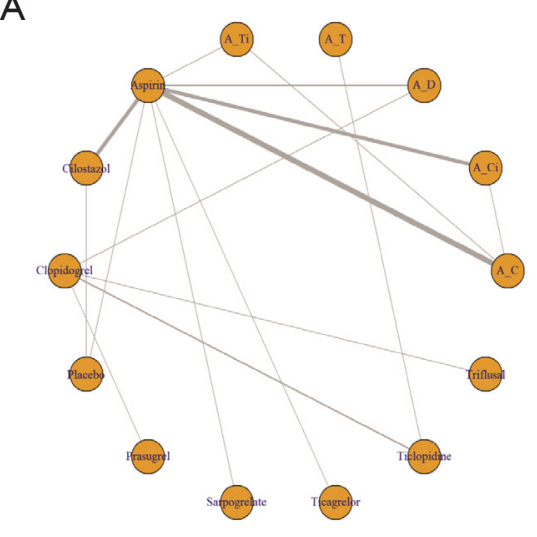

D

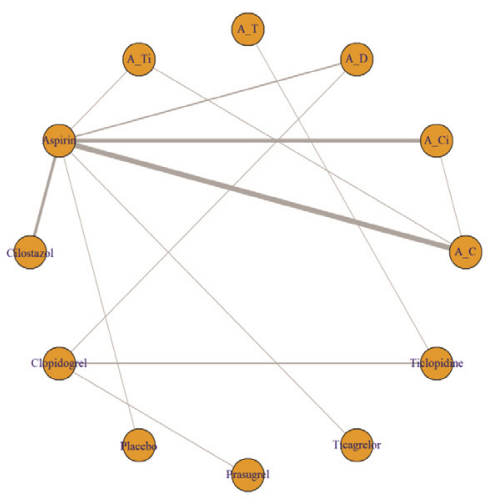

B

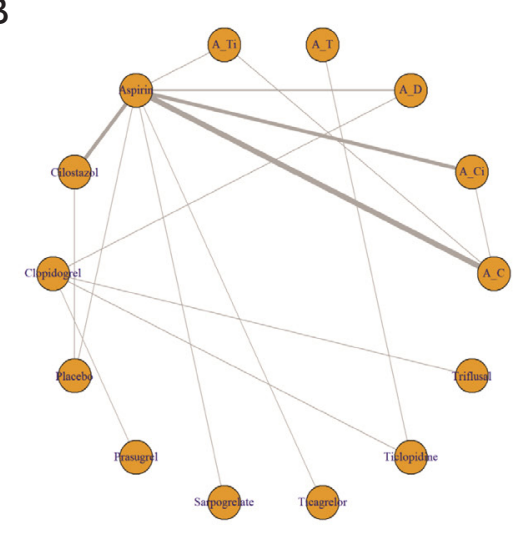

E

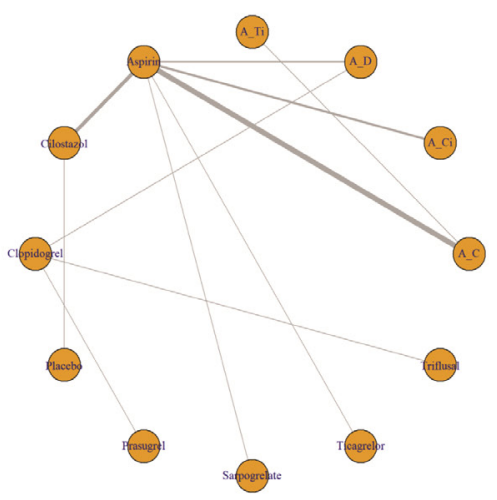

C

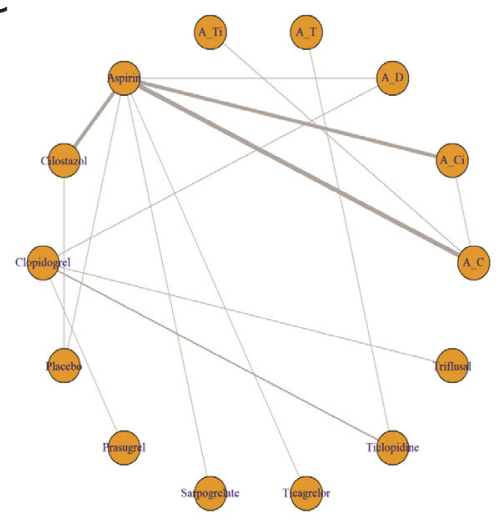

Figure 2 Network plots for the antiplatelet regimens. (A) Recurrent stroke; (B) recurrent ischemic stroke; (C) composite vascular events; (D) major bleeding; (E) all bleeding. A_Ti, aspirin plus ticagrelor; A_T, aspirin plus ticlopidine; A_D, aspirin plus dipyridamole; A_C, aspirin plus clopidogrel; A_Ci, aspirin plus cilostazol.

$(\mathrm{RR}=0.37,95 \%$ CrI: $0.18-0.69)$ was associated with a lower risk of major bleeding (Table S3); cilostazol was the first, and aspirin plus ticagrelor was the last (Table S4 and Figure S3).

\section{All bleeding}

Twenty-four trials, with a sample size of 50,325, reported all forms of bleeding. In Figure 3, most antiplatelet regimens were not associated with a significantly higher risk of all bleeding than the placebo, excluding aspirin plus ticagrelor $(\mathrm{RR}=3.89,95 \% \mathrm{CrI}: 1.54-10.59)$ and aspirin plus clopidogrel ( $\mathrm{RR}=2.48$, 95\% CrI: $1.10-5.81)$. When compared with aspirin, aspirin plus ticagrelor $(\mathrm{RR}=2.41$, 95\% CrI: $1.38-4.43$ ) and aspirin plus clopidogrel (RR $=1.52$, 95\% CrI: $1.12-2.13$ ) were associated with a higher risk of all bleeding, whereas cilostazol ( $\mathrm{RR}=0.64,95 \%$ CrI: 0.47-0.80) was associated with a lower risk (Table S3); cilostazol was ranked first, and aspirin plus ticagrelor was ranked last (Table S4 and Figure S3).

\section{Ranking and NCB}

A clustered three-dimensional rank plot demonstrated that cilostazol was the best antiplatelet therapy based on the NCB in relation to recurrent strokes, major bleeding, and composite vascular events (Figure 4A).

\section{Inconsistency assessment and publication bias}

Figure S4 shows the inconsistencies between the direct and indirect comparisons. There was no evidence of inconsistencies between the effect estimates of the direct and indirect evidence, except for those for recurrent IS in the aspirin $v s$. aspirin plus ticagrelor group $(\mathrm{P}=0.046)$. This assessment could not be performed for all bleeding due to a lack of outcome data. Symmetric funnel plots showed that there was no evidence of 
A

Compared with Placebo
Aspirin+Clopidogrel
Aspirin+Cilostazol
Aspirin+Dipyridamole
Aspirin+Ticlopidine
Aspirin+Ticagrelor
Aspirin
Cilostazol
Clopidogrel
Prasugrel
Sarpogrelate
Ticagrelor
Ticlopidine
Triflusal

C

Compared with Place bo Aspirin+Clopidogrel Aspirin+Cilostazol Aspirin+Dipyridamole Aspirin+Ticlopidine Aspirin+Ticagrelor Aspirin Cilostazol Clopidogrel Prasugrel Sarpogrelate Ticagrelor Ticlopidine Triflusal

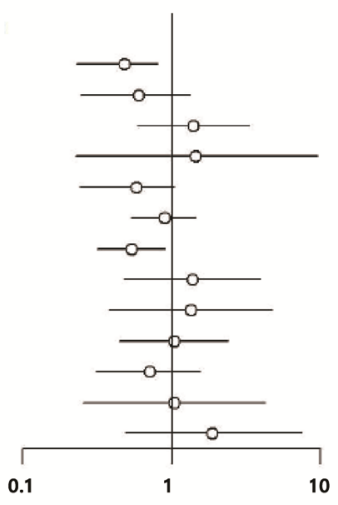

Relative risk (95\% Crl)

$0.53(0.27,0.83)$ $0.64(0.29,1.28)$ $1.33(0.63,2.85)$ $1.38(0.27,7.02)$ $0.62(0.28,1.03)$ $0.91(0.58,1.38)$ $0.58(0.36,0.91)$ $1.31(0.52,3.28)$ $1.30(0.43,3.85)$ $1.03(0.49,2.14)$ $0.74(0.36,1.47)$ $1.03(0.30,3.52)$ $1.73(0.53,5.69)$

Relative risk (95\% Crl)

$0.56(0.37,0.74)$ $0.67(0.38,1.15)$ $0.91(0.55,1.45)$ $0.83(0.26,2.74)$ $0.38(0.19,0.77)$ $0.86(0.65,1.08)$ $0.61(0.45,0.80)$ $0.91(0.51,1.56)$ $0.88(0.44,1.72)$ $0.91(0.57,1.42)$ $0.70(0.46,1.05)$ $0.81(0.39,1.64)$ $1.28(0.56,2.94)$
B

Compared with Place bo
Aspirin+Clopidogrel
Aspirin+Cilostazol
Aspirin+Dipyridamole
Aspirin+Ticlopidine
Aspirin+Ticagrelor
Aspirin
Cilostazol
Clopidogrel
Prasugrel
Sarpogrelate
Ticagrelor
Ticlopidine
Triflusal

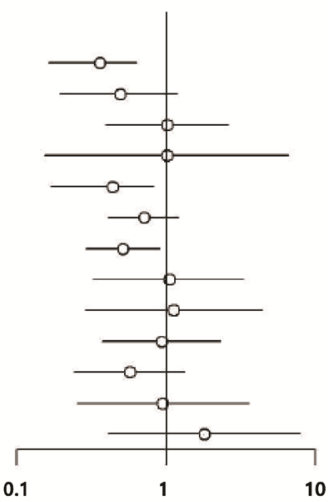

Relative risk (95\% Crl) $0.41(0.20,0.67)$ $0.54(0.24,1.15)$ $1.01(0.44,2.30)$ $1.01(0.19,5.15)$ $0.48(0.21,0.84)$ $0.74(0.46,1.18)$ $0.56(0.34,0.90)$ $1.04(0.37,2.82)$ $1.10(0.33,3.63)$ $0.94(0.42,2.06)$ $0.61(0.28,1.28)$ $0.95(0.30,3.00)$ $1.66(0.45,6.04)$
Compared with Place bo Aspirin+Clopidogrel Aspirin+Cilostazol Aspirin+Dipyridamole Aspirin+Ticagrelor Aspirin Cilostazol

Clopidogrel Prasugrel Ticagrelor Ticlopidine Aspirin+Ticlopidine

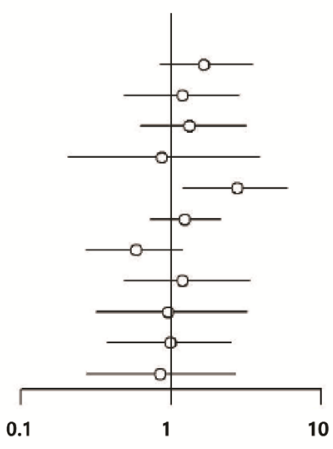

Relative risk (95\% Crl)

$1.91(0.78,5.00)$ $1.23(0.38,3.85)$ $1.44(0.54,4.43)$ $0.82(0.12,5.81)$ $3.74(1.24,10.17)$ $1.31(0.65,2.69)$ $0.49(0.18,1.23)$ $1.25(0.38,4.80)$ $0.94(0.22,4.52)$ $0.97(0.27,3.28)$ $0.79(0.18,3.56)$
E

Compared with Placebo Aspirin+Clopidogrel Aspirin+Cilostazol Aspirin+Dipyridamole Aspirin+Ticagrelor

Aspirin

Cilostazol

Clopidogrel

Prasugrel

Sarpogrelate

Ticagrelor

Triflusal

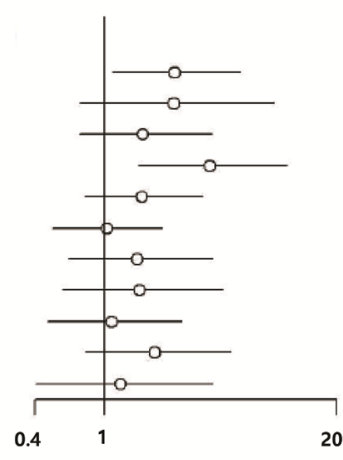

Relative risk (95\% Crl)

$2.48(1.10,5.81)$

$2.44(0.72,8.93)$

$1.64(0.72,4.01)$

$3.89(1.54,10.59)$

$1.61(0.77,3.56)$

$1.03(0.50,2.11)$

$1.51(0.62,4.05)$

$1.57(0.57,4.61)$

$1.10(0.47,2.71)$

$1.90(0.77,5.10)$

$1.23(0.41,4.05)$

Figure 3 Forrest plots for the antiplatelet regimens and placebo. (A) Recurrent stroke; (B) recurrent ischemic stroke; (C) composite vascular events; (D) major bleeding; (E) all bleeding.

publication bias in this NMA (Figure S5).

\section{Subgroup analysis}

\section{Before 72 hours from stroke onset}

Fourteen trials were included in this analysis. Most of the studies compared dual antiplatelet therapy (DAPT) and monotherapy, and the studies on monotherapy were Chen (10) for aspirin vs. placebo, Lee et al. (13) for cilostazol and aspirin, and Wang et al. (20) for ticagrelor and aspirin. The durations of treatment with aspirin plus dipyridamole and aspirin plus cilostazol were not limited. The durations 

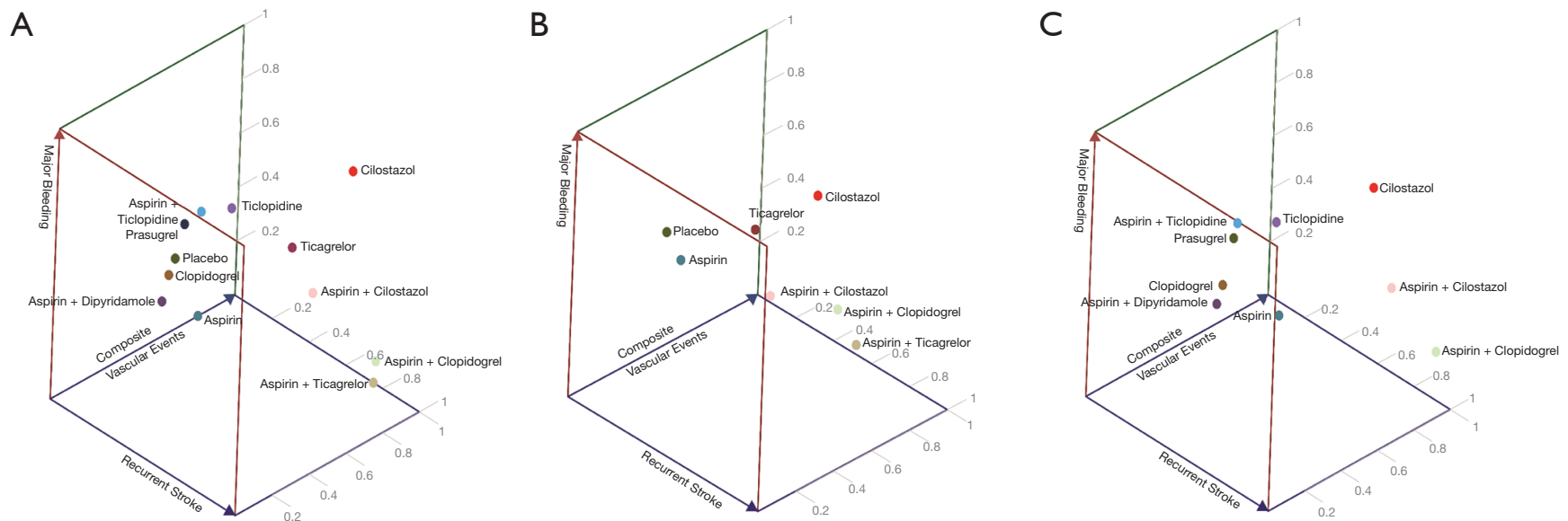

Figure 4 Three-dimensional clustered ranking plots. The $\mathrm{x}, \mathrm{y}$, and z-axes show the surface under the cumulative ranking curve (SUCRA) values for recurrent stroke, composite vascular events, and major bleeding, respectively. The point in the upper right is a hypothetical point with $100 \%$ SUCRA values for recurrent stroke, composite vascular events, and major bleeding. The antiplatelet regimen with ranking closest to this hypothetical point can be considered to have the greatest net clinical benefit. (A) Entire population; (B) seventy-two hours before stroke onset; (C) seventy-two hours after stroke onset.

of treatment with aspirin plus clopidogrel and aspirin plus ticagrelor were limited to three weeks and one month.

The antiplatelet regimens showed no significant differences in the risks of all outcomes compared with placebo (Figure S6). However, the aspirin plus clopidogrel combination was associated with lower risks of recurrent stroke, composite vascular events, and recurrent IS than aspirin $[\mathrm{RR}=0.59,95 \%$ CrI: (0.30-0.93), 0.63 (0.36-0.89), and $0.54(0.25-0.90)]$. Conversely, aspirin plus ticagrelor and aspirin plus clopidogrel were associated with a higher risk of all bleeding than aspirin $(\mathrm{RR}=2.41,95 \% \mathrm{CrI}$ : 1.01-6.17; RR $=1.52,95 \%$ CrI: $1.01-2.47)$, although these two DAPTs were not associated with a significantly higher risk of major bleeding than placebo or aspirin. Although cilostazol did not show significantly different safety and efficacy from placebo or aspirin, its SUCRA rankings were first, second, and third for major bleeding, recurrent stroke, and composite vascular events, respectively, and it had the high NCB (Figure S7 and Figure 4B).

\section{After 72 hours from stroke onset}

Eighteen trials were included. None of the included trials excluded patients with index events within 72 hours from the symptom onset to the treatment. Nevertheless, most of the index events developed 72 hours after symptom onset, and most studies had follow-ups lasting for three or more months, which reflected the secondary prevention of the chronic and stable stages compared with other subgroups.

The aspirin plus clopidogrel combination was associated with lower risks of recurrent stroke and recurrent IS than the placebo in the subgroup analysis 72 hours after stroke onset ( $\mathrm{RR}=0.26,95 \% \mathrm{CrI}$ : 0.09-0.75; RR $=0.23$, 95\% CrI: 0.08-0.66). Cilostazol was also associated with a lower risk of recurrent stroke than placebo $(\mathrm{RR}=0.52,95 \% \mathrm{CrI}$ : 0.28-0.99). The outcomes of the aspirin plus cilostazol combination treatment were not significantly different from those of the aspirin or placebo treatment, but its SUCRA rankings were second for recurrent stroke, recurrent IS, and composite vascular events. The detailed relative risks, 95\% CrI, and SUCRA rankings for all the outcomes are provided in Figure S8 and Figure S9.

Regarding safety, cilostazol was associated with lower risks of major and all bleeding than aspirin $(\mathrm{RR}=0.36$, 95\% CrI: 0.10-0.95; RR =0.59, 95\% CrI: 0.23-0.97, respectively). As with NMA, cilostazol had the highest $\mathrm{NCB}$ (Figure 4C).

\section{Discussion}

This was the first systematic review and NMA to comparatively assess the efficacy and safety of antiplatelet regimens for the secondary prevention of non-cardioembolic IS or TIA in Asian populations, and it enrolled 84,103 patients from 32 trials. Based on the primary efficacy 
outcome, aspirin plus clopidogrel and cilostazol were associated with a lower risk of recurrent stroke than placebo. Based on the primary safety outcome, most antiplatelet regimens, excluding aspirin plus ticagrelor, were not associated with a higher risk of major bleeding; only cilostazol was associated with a lower risk of major bleeding than aspirin. Finally, the clustered three-dimensional rank plot demonstrated that cilostazol, among the antiplatelet regimens, had the highest NCB for all the main outcomes.

The risk of recurrent stroke is higher during the acute than the chronic period. To reduce the risk of recurrent stroke, potent antiplatelet agents were administered as early as possible. Similar to the results of major clinical trials, our subgroup analysis (symptom onset-to-treatment $<72$ hours) showed that aspirin plus clopidogrel and aspirin plus ticagrelor had the highest ranking for recurrent stroke, composite vascular outcomes, and recurrent IS based on the SUCRA. Although the treatment durations for these two DAPTs were within one month in all the included trials, the risks of bleeding of all forms, as well as major bleeding, were also higher; this can be partially attributed to the higher bleeding risk in Asians. In the main analysis, these two DAPTs were associated with increased risks of all bleeding; aspirin plus ticagrelor, especially, increased the risk of major bleeding, which should be considered when determining a long-term secondary prevention strategy.

For long-term secondary prevention of stroke, aspirin is considered the standard drug, and most guidelines recommend it $(3,42)$. However, aspirin has been investigated mainly in Western countries, and several meta-analyses have been performed based on results from Western populations $(1,43)$. The effect of aspirin has not been thoroughly investigated in the Asian population. Therefore, several Western trials that reported a good efficacy of aspirin were excluded from our NMA. This seems to be the main reason why the efficacy of aspirin was not better than that of placebo in our NMA. In addition, a lower dose of aspirin was mainly used in contrast with the moderate- to high-dose aspirin used in Asian populations with concerns of bleeding (44). Clopidogrel did not also show a significantly better efficacy than placebo in our NMA. Clopidogrel is a prodrug, which has to be converted into an active metabolite by CYP2C19 to inhibit platelet function. Because of the high prevalence of CYP2C19 polymorphism (poor metabolizer) in Asian populations (45), clopidogrel does not seem to show efficacy comparable to that in Western populations.

Cilostazol has multiple actions that affect various factors associated with thrombus formation and vascular occlusion, such as increasing nitric oxide (an endogenous vasodilatation factor), decreasing intracellular calcium concentration, and inhibiting the proliferation of smooth muscle cells (46). Therefore, cilostazol can reduce the risk of stroke in those with small-vessel disease prone to intracerebral hemorrhage and decrease the atherosclerotic burden in patients with intracranial atherosclerosis. Cilostazol protects all components of the blood-brain barrier, including the endothelial cells, pericytes, tight junction proteins, adherence junction proteins, and the basement membrane, suggesting that it also reduces hemorrhagic stroke (47). Furthermore, the reversible platelet inhibition mechanism enables a relatively rapid recovery time of platelet function and low bleeding risk (48). Genetic polymorphisms of CYP450 also affect cilostazol metabolism (49), but the influence on cilostazol is limited in Asia because of the low incidence of polymorphisms related to poor-metabolizers (50). These findings are consistent with our finding that cilostazol was associated with lower infarction and bleeding risks than other antiplatelet agents, given the prevalence of stroke and the high risk of bleeding in Asians. Interestingly, a recent study reported that a cilostazol-based combination with aspirin or clopidogrel was more efficacious in reducing IS than a single antiplatelet agent (aspirin or clopidogrel) without increasing the risk of hemorrhage (51). However, due to a lack of data on the separate outcomes, our NMA did not include this recent study, and we could have underestimated the efficacy and safety of the aspirin plus cilostazol combination (52). Further large-sample randomized trials of DAPT based on cilostazol are warranted.

This systematic review has several limitations. First, most of the antiplatelet agents did not show significantly better efficacies than placebo in our NMA. As mentioned above, the most important trials that tested aspirin's efficacy were excluded because they were conducted in Western countries. Therefore, aspirin was not significantly more efficacious than placebo in our NMA. Second, this is a study-level meta-analysis that lacked individual patient data. Although this NMA included trials with various durations of follow-up, we used relative RRs rather than hazard ratios, due to the deficient individual patient data. However, because all studies with follow-up durations within one month were included in the "before 72 hours from stroke onset" subgroup, the effect of the follow-up duration diversity may have been restricted to an extent during the subgroup analyses. Third, there was an inconsistency 
between the direct and indirect evidence for the effects of aspirin and aspirin plus ticagrelor on recurrent IS. This inconsistency resolved during the subgroup analysis. Therefore, the increased heterogeneity was thought to originate from the differences in the durations from the symptom onset to treatment. Considering that there were no differences between the outcomes of the primary and subgroup analyses, the overall consistency is thought to have been satisfactory, meaning that our network model selection was appropriate. Fourth, although most included trials included Asian populations, two global trials $(23,29)$ only involved $32 \%$ and $42 \%$ of Asians, respectively; one trial (20) used a subgroup analysis from a global trial, which is SOCRATES (53). All the populations used for our NMA were not purely Asian, and the subgroup analysis has a risk of randomization error. Finally, the mechanism of IS and the duration of treatment with DAPT were not considered, although they can influence the antiplatelet treatment strategy for secondary prevention.

In conclusion, this Bayesian NMA indicates that cilostazol is a better choice than other antiplatelet regimens for Asians with non-cardioembolic stroke or TIA, based on the efficacy and safety outcomes. The selection of appropriate antiplatelet agents may differ with the riskbenefit assessment outcome and the duration between symptom onset and treatment. Due to the limitations of this NMA, further head-to-head randomized trials are needed to determine the appropriate antiplatelet regimens for various clinical situations.

\section{Acknowledgments}

The authors would like to thank Eun-Ji Kang from the Medical Library of Korea University for searching abstracts and articles related to this study and Editage (www.editage. co.kr) for the English language editing.

Funding: This research was supported by the Basic Science Research Program through the National Research Foundation of Korea (NRF) funded by the Ministry of Science and ICT (NRF-2020R1C1C1009294).

\section{Footnote}

Reporting Checklist: The authors have completed the PRISMA NMA reporting checklist. Available at http:// dx.doi.org/10.21037/atm-20-7951

Conflicts of Interest: All authors have completed the ICMJE uniform disclosure form (available at http://dx.doi. org/10.21037/atm-20-7951). JMJ serves as an unpaid editorial board member of Annals of Translational Medicine from Nov 2019 to Oct 2021. JMJ currently serves on the Editorial Board of Neurology for this journal, JMJ reports other from Pfizer Pharmaceutical Company, other from Sanofi-Aventis Pharmaceutical Company, other from Daewoong Pharmaceutical Company, other from Otuska, other from Hanmi Pharmaceutical Company, outside the submitted work. The other authors have no conflicts of interest to declare.

Ethical Statement: The authors are accountable for all aspects of the work in ensuring that questions related to the accuracy or integrity of any part of the work are appropriately investigated and resolved.

Open Access Statement: This is an Open Access article distributed in accordance with the Creative Commons Attribution-NonCommercial-NoDerivs 4.0 International License (CC BY-NC-ND 4.0), which permits the noncommercial replication and distribution of the article with the strict proviso that no changes or edits are made and the original work is properly cited (including links to both the formal publication through the relevant DOI and the license). See: https://creativecommons.org/licenses/by-nc-nd/4.0/.

\section{References}

1. Antiplatelet Trialists' Collaboration. Collaborative overview of randomised trials of antiplatelet therapy-I: Prevention of death, myocardial infarction, and stroke by prolonged antiplatelet therapy in various categories of patients. BMJ 1994;308:81-106.

2. Baigent C, Blackwell L, Collins R, et al. Aspirin in the primary and secondary prevention of vascular disease: collaborative meta-analysis of individual participant data from randomised trials. Lancet 2009;373:1849-60.

3. Kernan WN, Ovbiagele B, Black HR, et al. Guidelines for the prevention of stroke in patients with stroke and transient ischemic attack: a guideline for healthcare professionals from the American Heart Association/ American Stroke Association. Stroke 2014;45:2160-2236.

4. Ueshima H, Sekikawa A, Miura K, et al. Cardiovascular disease and risk factors in Asia: a selected review. Circulation 2008;118:2702-9.

5. Gorelick PB, Wong KS, Bae HJ, et al. Large artery intracranial occlusive disease: a large worldwide burden 
but a relatively neglected frontier. Stroke 2008;39:2396-9.

6. Levine GN, Jeong YH, Goto S, et al. Expert consensus document: World Heart Federation expert consensus statement on antiplatelet therapy in East Asian patients with ACS or undergoing PCI. Nat Rev Cardiol 2014;11:597-606.

7. Hooi JKY, Lai WY, Ng WK, et al. Global prevalence of helicobacter pylori infection: Systematic review and metaanalysis. Gastroenterology 2017;153:420-9.

8. Moher D, Liberati A, Tetzlaff J, et al. Preferred reporting items for systematic reviews and meta-analyses: The PRISMA statement. BMJ 2009;339:b2535.

9. Higgins JP, Thomas J, Chandler J, et al. Cochrane handbook for systematic reviews of interventions. 2nd ed. Chichester (UK): John Wiley \& Sons, 2019:117-227.

10. Chen ZM. CAST: randomised placebo-controlled trial of early aspirin use in 20,000 patients with acute ischaemic stroke. Lancet 1997;349:1641-9.

11. Chairangsarit $\mathrm{P}$, Sithinamsuwan $\mathrm{P}$, Niyasom S, et al. Comparison between aspirin combined with dipyridamole versus aspirin alone within 48 hours after ischemic stroke event for prevention of recurrent stroke and improvement of neurological function: a preliminary study. J Med Assoc Thai 2005;88:S148-54.

12. Wong KS, Chen C, Fu J, et al. Clopidogrel plus aspirin versus aspirin alone for reducing embolisation in patients with acute symptomatic cerebral or carotid artery stenosis (CLAIR study): a randomised, open-label, blindedendpoint trial. Lancet Neurol 2010;9:489-97.

13. Lee YS, Bae HJ, Kang DW, et al. Cilostazol in Acute Ischemic Stroke Treatment (CAIST trial): a randomized double-blind non-inferiority trial. Cerebrovasc Dis 2011;32:65-71.

14. Nakamura T, Tsuruta S, Uchiyama S. Cilostazol combined with aspirin prevents early neurological deterioration in patients with acute ischemic stroke: a pilot study. J Neurol Sci 2012;313:22-6.

15. Wang Y, Wang Y, Zhao X, et al. Clopidogrel with aspirin in acute minor stroke or transient ischemic attack. N Engl J Med 2013;369:11-9.

16. Yi X, Lin J, Wang C, et al. A comparative study of dual versus monoantiplatelet therapy in patients with acute large-artery atherosclerosis stroke. J Stroke Cerebrovasc Dis 2014;23:1975-81.

17. He F, Xia C, Zhang JH, et al. Clopidogrel plus aspirin versus aspirin alone for preventing early neurological deterioration in patients with acute ischemic stroke. J Clin
Neurosci 2015;22:83-6.

18. Yi X, Chi W, Wang C, et al. Low-molecular-weight heparin or dual antiplatelet therapy is more effective than aspirin alone in preventing early neurological deterioration and improving the 6-month outcome in ischemic stroke patients. J Clin Neurol 2015;11:57-65.

19. Hong KS, Lee SH, Kim EG, et al. Recurrent Ischemic Lesions After Acute Atherothrombotic Stroke: Clopidogrel Plus Aspirin Versus Aspirin Alone. Stroke 2016;47:2323-30.

20. Wang Y, Minematsu K, Wong KS, et al. Ticagrelor in Acute Stroke or Transient Ischemic Attack in Asian Patients: From the SOCRATES Trial (Acute Stroke or Transient Ischemic Attack Treated With Aspirin or Ticagrelor and Patient Outcomes). Stroke 2017;48:167-73.

21. Aoki J, Iguchi Y, Urabe T, et al. Acute Aspirin Plus Cilostazol Dual Therapy for Noncardioembolic Stroke Patients Within 48 Hours of Symptom Onset. J Am Heart Assoc 2019;8:e012652.

22. Wang Y, Chen W, Lin Y, et al. Ticagrelor plus aspirin versus clopidogrel plus aspirin for platelet reactivity in patients with minor stroke or transient ischaemic attack: open label, blinded endpoint, randomised controlled phase II trial. BMJ 2019;365:12211.

23. Johnston SC, Amarenco P, Denison H, et al. Ticagrelor and Aspirin or Aspirin Alone in Acute Ischemic Stroke or TIA. N Engl J Med 2020;383:207-17.

24. Gotoh F, Tohgi H, Hirai S, et al. Cilostazol stroke prevention study: a placebo-controlled double-blind trial for secondary prevention of cerebral infarction. J Stroke Cerebrovasc Dis 2000;9:147-57.

25. Ito E, Takahashi A, Yamamoto H, et al. Ticlopidine alone versus ticlopidine plus aspirin for preventing recurrent stroke. Intern Med 2003;42:793-9.

26. Kwon SU, Cho YJ, Koo JS, et al. Cilostazol prevents the progression of the symptomatic intracranial arterial stenosis: the multicenter double-blind placebo-controlled trial of cilostazol in symptomatic intracranial arterial stenosis. Stroke 2005;36:782-6.

27. Huang Y, Cheng Y, Wu J, et al. Cilostazol as an alternative to aspirin after ischaemic stroke: a randomised, doubleblind, pilot study. Lancet Neurol 2008;7:494-9.

28. Fukuuchi Y, Tohgi H, Okudera T, et al. A randomized, double-blind study comparing the safety and efficacy of clopidogrel versus ticlopidine in Japanese patients with noncardioembolic cerebral infarction. Cerebrovasc Dis 2008;25:40-9. 
29. Sacco RL, Diener HC, Yusuf S, et al. Aspirin and extended-release dipyridamole versus clopidogrel for recurrent stroke. N Engl J Med 2008;359:1238-51.

30. Shinohara Y, Nishimaru K, Sawada T, et al. SarpogrelateAspirin Comparative Clinical Study for Efficacy and Safety in Secondary Prevention of Cerebral Infarction (S-ACCESS): A randomized, double-blind, aspirincontrolled trial. Stroke 2008;39:1827-33.

31. Guo JJ, Xu E, Lin QY, et al. Effect of cilostazol on cerebral arteries in secondary prevention of ischemic stroke. Neurosci bull 2009;25:383-90.

32. Uchiyama S, Fukuuchi Y, Yamaguchi T. The safety and efficacy of clopidogrel versus ticlopidine in Japanese stroke patients: combined results of two Phase III, multicenter, randomized clinical trials. J Neurol 2009;256:888-97.

33. Shinohara Y, Katayama Y, Uchiyama S, et al. Cilostazol for prevention of secondary stroke (CSPS 2): an aspirincontrolled, double-blind, randomised non-inferiority trial. Lancet Neurol 2010;9:959-68.

34. Uchiyama S, Ikeda Y, Urano Y, et al. The Japanese aggrenox (extended-release dipyridamole plus aspirin) stroke prevention versus aspirin programme (JASAP) study: a randomized, double-blind, controlled trial. Cerebrovasc Dis 2011;31:601-13.

35. Kwon SU, Hong KS, Kang DW, et al. Efficacy and safety of combination antiplatelet therapies in patients with symptomatic intracranial atherosclerotic stenosis. Stroke 2011;42:2883-90.

36. Han SW, Lee SS, Kim SH, et al. Effect of cilostazol in acute lacunar infarction based on pulsatility index of transcranial doppler (ECLIPse): a multicenter, randomized, double-blind, placebo-controlled trial. Eur Neurol 2013;69:33-40.

37. Uchiyama S, Sakai N, Toi S, et al. Final Results of Cilostazol-Aspirin Therapy against Recurrent Stroke with Intracranial Artery Stenosis (CATHARSIS). Cerebrovasc Dis Extra 2015;5:1-13.

38. Han SW, Kim YJ, Ahn SH, et al. Effects of triflusal and clopidogrel on the secondary prevention of stroke based on cytochrome p450 2c19 genotyping. J Stroke 2017;19:356-64.

39. Zuo FT, Liu H, Wu HJ, et al. The effectiveness and safety of dual antiplatelet therapy in ischemic cerebrovascular disease with intracranial and extracranial arteriostenosis in Chinese patients: a randomized and controlled trial. Medicine (Baltimore) 2017;96:e5497.

40. Kim BJ, Lee EJ, Kwon SU, et al. Prevention of cardiovascular events in asian patients with ischaemic stroke at high risk of cerebral haemorrhage (PICASSO): amulticentre, randomised controlled trial. Lancet Neurol 2018;17:509-18.

41. Ogawa A, Toyoda K, Kitagawa K, et al. Comparison of prasugrel and clopidogrel in patients with noncardioembolic ischaemic stroke: a phase 3, randomised, non-inferiority trial (PRASTRO-I). Lancet Neurol 2019;18:238-47.

42. Wein T, Lindsay MP, Côté R, et al. Canadian stroke best practice recommendations: Secondary prevention of stroke, sixth edition practice guidelines, update 2017. Int J Stroke 2018;13:420-43.

43. Algra A, van Gijn J. Cumulative meta-analysis of aspirin efficacy after cerebral ischaemia of arterial origin. J Neurol Neurosurg Psychiatry 1999;66:255.

44. Goto S, Ikeda Y, Chan JC, et al. Risk-factor profile, drug usage and cardiovascular events within a year in patients with and at high risk of atherothrombosis recruited from Asia as compared with those recruited from non-Asian regions: a substudy of the REduction of Atherothrombosis for Continued Health (REACH) registry. Heart Asia 2011;3:93-8.

45. Rosemary J, Adithan C. The pharmacogenetics of CYP2C9 and CYP2C19: ethnic variation and clinical significance. Curr Clin Pharmacol 2007;2:93-109.

46. Noma K, Higashi Y. Cilostazol for treatment of cerebral infarction. Expert Opin Pharmacother 2018;19:1719-26.

47. Takagi T, Hara H. Protective effects of cilostazol against hemorrhagic stroke: Current and future perspectives. J Pharmacol Sci 2016;131:155-61.

48. Goto S. Cilostazol: potential mechanism of action for antithrombotic effects accompanied by a low rate of bleeding. Atheroscler Suppl 2005;6:3-11.

49. Yoo HD, Cho HY, Lee YB. Population pharmacokinetic analysis of cilostazol in healthy subjects with genetic polymorphisms of CYP3A5, CYP2C19 and ABCB1. Br J Clin Pharmacol 2010;69:27-37.

50. Lee SJ, Usmani KA, Chanas B, et al. Genetic findings and functional studies of human CYP3A5 single nucleotide polymorphisms in different ethnic groups. Pharmacogenetics 2003;13:461-72.

51. Toyoda K, Uchiyama S, Yamaguchi T, et al. Dual antiplatelet therapy using cilostazol for secondary prevention in patients with high-risk ischaemic stroke in Japan: a multicentre, open-label, randomised controlled trial. Lancet Neurol 2019;18:539-48. 
52. Kim SM, Jung JM, Kim BJ, et al. Cilostazol Mono and Combination Treatments in Ischemic Stroke: An Updated Systematic Review and Meta-analysis. Stroke 2019;50:3503-11.

Cite this article as: Jung SJ, Shim SR, Kim BJ, Jung JM. Antiplatelet regimens for Asian patients with ischemic stroke or transient ischemic attack: a systematic review and network meta-analysis. Ann Transl Med 2021;9(9):753. doi: 10.21037/ atm-20-7951
53. Johnston SC, Amarenco P, Albers GW, et al. Ticagrelor versus Aspirin in Acute Stroke or Transient Ischemic Attack. N Engl J Med 2016;375:35-43. 
Table S1 Search strategy

Search Medline

\#1 Stroke, Lacunar[mh] OR lacunar stroke*tiab] OR lacunar infarct"tiab] OR lacunar syndrome*ttiab] OR ischemic stroke* [tiab] OR ischaemic stroke*ttiab] OR ischemic brain stroke*t[tiab] OR ischaemic brain stroke*t[tiab] OR cerebrovascular ischemia*tiab] $\mathrm{OR}$ cerebrovescular ischemia*tiab] OR ischemic braintiab] OR ischeemic brain[tiab] OR ischemic encephalopath* [tiab] OR ischaemicencephalopathttiab]

\#2 Cerebral Infarction[mh] OR brain infarct"tiab] OR brain stem infarct"ttiab] OR cerebral infarct ttiab] OR

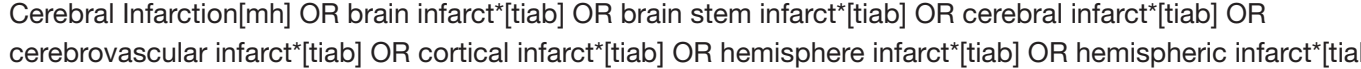
Ischemic Attack, Transient[mh] OR transient ischemic attack ${ }^{*}$ tiab] OR transient ischaemic attack $k^{*}$ tiab] OR

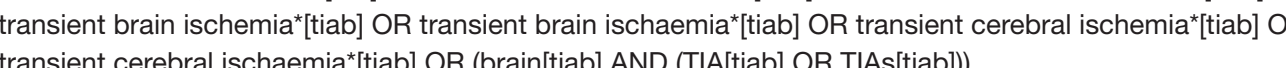

\#4 \#1 OR\#2 OR\#3

\#5 Platelet Aggregation Inhibitors[mh] OR platelet aggregation inhibitor"[tiab] OR platelet antiaggregant"[tiab] OR platelet anti-aggregant* [tiab] OR platelet inhibitorkttiab] OR antiplatelet agent"tiab] OR antiplatelet drug*[tiab] OR anti-platelet agent" [tiab] OR anti-platelet drug*tiab] OR platelet antagonist"titiab] OR antithrombotic agent"tiab] OR anti-thrombotic agent" $[$ tiab] OR thrombocyte aggregation inhibitor" [tiab]

\#6 Aspirin[mh] OR aspirin[tw] OR acetylsalicylic acid[tw] OR acetyl salicylic acid[tw] OR acetosalicylic acid[tw] OR Acylpyrin[tw] OR Colfaritttw] OR Ecotrin[tw] OR Endosprin[tw] OR Magnecyl[tw] OR Micristin[tw] OR Polopirin[tw OR Polopiryna[tw] OR Solupsan[tw] OR Zorprin[tw] OR Acetysal[tw] OR Aloxiprimum[tw] OR Dispril[tw] OR Easprin[tw] OR Solprin[tw]

\#7 Clopidogrel[mh] OR Clopidogrel[tw] OR SC 25989C[tw] OR SC 25990C[tw] OR SR 25989[tw] OR Iscover[tw] OR PCR-4099[tw] OR Plavix[tw]

\#8 Cilostazol[mh] OR Cilostazolltw] OR OPC-13013[tw] OR Pletalltw] OR pletaal[tw]

\#9 Ticagrelor[mh] OR Ticagrelor[tw] OR Brilique[tw] OR AZD 6140[tw] OR Brilinta[tw]

\#10 Prasugrel Hydrochloride[mh] OR Prasugrelltw] OR CS 747[tw] OR Efient[tw] OR Effient[tw] OR LY 640315[tw]

\#11 triflusalltw] OR 2-acetoxy-4-trifluoromethylbenzoic acid[tw] OR Disgren[tw] OR tecnosal[tw] OR triflux[tw] OR aflen[tw]

\#12 Dipyridamole[mh] OR Dipyridamole[tw] OR Dipyramidole[tw] OR Cerebrovase[tw] OR Persantine[tw] OR Persantin[tw] OR Curati[WW OR Curaty[tw] OR Kurantiltw] OR Miosen[tw] OR Novo-Dipirdol[tw] OR Antistenocardin[tw] OR Cléridium[tw] OR Cleridium[tw]

$\# 13 \quad$ OR \#5 to \#12

\#14 Secondary Prevention[mh] OR secondary prevention*tww] OR secondary disease prevention*tww] OR relapse prevention"[tw] OR recurrence prevention ${ }^{*}[\mathrm{tw}]$

\#4 AND \#13 AND \#14

(randomized controlled trial[pt] OR controlled clinical trial[ipt] OR randomized[tiab] OR placebo[tiab] OR clinical trials as topic[mh:noexp] OR randomly[tiab] OR trial[ti) NOT (animals[mh] NOT humans[mhh]

$\# 17 \quad \# 15$ AND \#16

\section{Embase}

(lacunar stroke'/de OR (lacunar NEXT/1 (stroke* OR infarct* OR syndrome*)):ti,ab OR (schn mic NEAR/3 stroke"):ti,ab OR (brain NEAR/3 isch* mit):ti,ab OR (cerebral NEAR/3 ncephalopath*):ti,ab)

('brain infarction'/exp OR (brain OR cerebral OR cerebrovascular OR cortical OR (hemis ${ }^{*}$ NEXT/2 infarct*)+itab)

('transient ischemic attack'/exp OR (transient NEAR/2 isch"mic NEAR/2 attack"):ti, b OR (transient NEAR/2 (brain OR cerebral) NEAR/2 ischtmit)itab OR (brain NEAR/5 OR T(As)::ti,ab)

$\# 1$ OR \#2 OR \#3

('antithrombotic agent'/de OR ((platelet OR thrombocyte) NEXT/2 (inhibitor* OR antiaggregant* $\mathrm{OR}$ anti-aggregant* ${ }^{*} \mathrm{OR}$ antagonist $\left.{ }^{*}\right)$ ):ti,ab OR ((antiplatelet OR antiplatelet OR antithrombotic OR anti-thrombotic) NEXT/2 (drug ${ }^{*}$ OR agent')):ti,ab)

('acetylsalicylic acid'/de OR (aspirin OR 'acetylsalicylic acid' OR 'acetyl salicylic

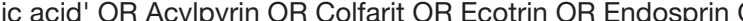
Magnecyl OR Micristin OR Polopirin OR Polopiryna OR Solupsan OR Zorprin OR Acetysal OR Aloxiprimum OR Dispril OR Easprin OR Solprin):ti,ab)

clopidogrel/de OR (Clopidogrel OR SC-25989C OR SC-25990C OR SR-25989 OR

cilostazol/de OR (Cilostazol OR OPC-13013 OR Pletal OR pletaal):ti,ab

ticagrelor/de OR (Ticagrelor OR Brilique OR AZD-6140 OR Brilinta):ti,ab

prasugrel/de OR (Prasugrel OR CS-747 OR Efient OR Effient OR LY-640315):ti,ab

triflusal/de OR (triflusal OR '2-acetoxy-4-trifluoromethylbenzoic acid' OR Disgren OR tecnosal OR triflux OR aflen):ti,ab

dipyridamole/de OR (Dipyridamole OR Dipyramidole OR Cerebrovase OR Persantine OR Persalln OR Guranti OR Cur (l)

OR 45 to $\# 12$

('secondary prevention'/de OR ((secondary OR relapse OR recurrence) NEXT/2 prevention*):ti,ab)

\#4 AND \#13 AND \#14

('randomized controlled trial'/de OR 'controlled clinical trial'/de OR randomi?ed:ti,ab OR placebo:ti,ab OR 'clinical trial (topic)'/de OR randomly:ti,ab OR trial:ti) NOT (anima OR placebo:ti,ab OR
exp NOT human/exp)

\#15 AND \#16

('conference paper'/exp OR 'conference paper'/it OR 'conference proceeding':pt OR 'conference review'/it OR 'conference abstract' it OR 'abstract report'/exp)

\#17 NOT \#18
Cochrane Trials

[mh "Stroke, Lacunar"] OR (lacunar NEXT (stroke* OR infarct* OR syndrome*)):ti,ab OR (schtmic NEAR/3 stroke*):ti,ab OR (brain NEAR/3 isch*mit):ti,ab OR (cerebral NEAR

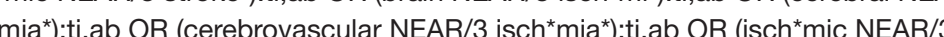
encephalopath*):ti,ab

[mh "Cerebral Infarction"] OR (brain OR cerebral OR cerebrovascular OR cortical OR (infarct):ti,ab

mh "Ischemic Attack, Transient"] OR (transient NEAR/2 isch*mic NEAR/2 attack"):ti,ab OR (transient NEAR/2 (brain OR cerebral) NEAR/2 isch*mia*):ti,ab OR (brain NEAR/5 (TIA OR TIAs): ti,ab

\#1 OR \#2 OR \#3

[mh "Platelet Aggregation Inhibitors"] OR ((platelet OR thrombocyte) NEXT/2 (inhibitor (a) platelet OR antithrombotic OR anti-thrombocytic) NEXT/2 (drug* OR agent")):ti,ab

[mh Aspirin] OR (aspirin OR "acetylsalicylic acid" OR "acetyl salicylic acid" OR "acetosalicylic acid" OR Acylpyrin OR Colfarit OR Ecotrin OR Endosprin OR Magnecy OR Micristin OR Polopirin OR Polopiryna OR Solupsan OR Zorprin OR Acetysal OR Aloxiprimum OR Dispril OR Easprin OR Solprin):ti,ab

Imh Clopidogrell OR (Clopidogrel OR SC-25

Imh Cilostazoll OR (Cilostazol OR OPC-13013 OR Pletal OR pletaal)'ti

Imh Ticagrelor] OR (Ticagrelor OR Brilique OR AZD-6140 OR Brilinta):ti,ab [mh "Prasugrel Hydrochloride"] OR (Prasugrel OR CS-747 OR Efient OR Effient OR LY640315):ti,ab

(triflusal OR "2-acetoxy-4-triffluoromethylbenzoic acid" OR Disgren OR tecnosal OR triflux OR aflen):ti,ab

[mh Dipyridamole] OR (Dipyridamole OR Dipyramidole OR Cerebrovase OR Persantine OR Antistenocardin OR Cléridium OR Cleridium):ti,ab

$\mathrm{OR} \# 5$ to \#12

[mh "Secondary Prevention"] OR ((secondary OR relapse OR recurrence) NEXT/2 prevention"):ti,ab

$\# 4$ AND \#13 AND \#14

Cochrane Reviews 10

Trials 343

Trials 


\begin{tabular}{|c|c|c|c|c|c|c|c|c|c|c|c|c|}
\hline Trial & Antiplatelet regimens & Asian & $F / U$ & $\mathrm{Tx}$ & Study & $\mathrm{N}$ & $\mathrm{T}$ & c & Male & Age & HTN & $\mathrm{DM}$ \\
\hline 1997 CAST, Chen ${ }^{10}$ & Aspirin vs. Placebo & $100 \%$ & $1 \mathrm{M}$ & $<48 \mathrm{~h}$ & IS & 20655 & 10335 & 10320 & $64 \%$ & 63 & $25 \%$ & NC \\
\hline 2000 CSPS, Gotoh ${ }^{11}$ & Cilostazol vs. Placebo & $100 \%$ & $22 \mathrm{M}$ & & IS & 1067 & 533 & 534 & $66 \%$ & 65 & $61 \%$ & $25 \%$ \\
\hline 2003 TOPALS, Ito ${ }^{12}$ & A_T vs. Ticlopidine & $100 \%$ & $19 \mathrm{M}$ & & $\mathrm{IS} / \mathrm{TIA}$ & 270 & 132 & 138 & $65 \%$ & 67 & $47 \%$ & $23 \%$ \\
\hline 2005 Chairangsarit $^{13}$ & A_D vs. Aspirin & $100 \%$ & $6 \mathrm{M}$ & $<48 \mathrm{~h}$ & is & 38 & 20 & 18 & $53 \%$ & 64 & $50 \%$ & $32 \%$ \\
\hline 2005 TOSS, Kwon ${ }^{14}$ & A_Ci vs. Aspirin & $100 \%$ & $6 \mathrm{M}$ & & is & 135 & 67 & 68 & $61 \%$ & 62 & $58 \%$ & $40 \%$ \\
\hline 2008 CASISP, Huang ${ }^{15}$ & Cilostazol vs. Aspirin & $100 \%$ & $15 \mathrm{M}$ & & is & 719 & 360 & 359 & $69 \%$ & 60 & $79 \%$ & $18 \%$ \\
\hline 2008 Fukuuchi $^{16}$ & Clopidogrel vs. Ticlopidine & $100 \%$ & $12 \mathrm{M}$ & & is & 1151 & 573 & 578 & $73 \%$ & 65 & $68 \%$ & $19 \%$ \\
\hline 2008 PRoFESS, Sacco ${ }^{17}$ & A_D vs. Clopidogrel & $32 \%$ & $30 \mathrm{M}$ & & is & 20332 & 10181 & 10151 & $64 \%$ & 66 & $74 \%$ & $29 \%$ \\
\hline 2008 S-ACCESS, Shinohara & Sarpogrelate vs. Aspirin & $100 \%$ & $19 \mathrm{M}$ & & IS & 1510 & 752 & 758 & $72 \%$ & 65 & $70 \%$ & $28 \%$ \\
\hline 2009 Guo $^{19}$ & Cilostazol vs. Aspirin & $100 \%$ & $12 \mathrm{M}$ & & is & 68 & 34 & 34 & $35 \%$ & 60 & NC & NC \\
\hline 2009 Uchiyama $^{20}$ & Clopidogrel vs. Ticlopidine & $100 \%$ & $12 \mathrm{M}$ & & is & 1869 & 941 & 928 & $72 \%$ & 64 & $70 \%$ & $22 \%$ \\
\hline 2010 CLAIR, Wong ${ }^{21}$ & A_C vs. Aspirin & $100 \%$ & $1 \mathrm{~W}$ & $<72 \mathrm{~h}$ & $\mathrm{IS} / \mathrm{TIA}$ & 98 & 46 & 52 & $78 \%$ & 59 & $64 \%$ & $38 \%$ \\
\hline 2010 CSPS2, Shinohara ${ }^{22}$ & Cilostazol vs. Aspirin & $100 \%$ & $29 \mathrm{M}$ & & is & 2757 & 1379 & 1378 & $72 \%$ & 63 & $74 \%$ & $29 \%$ \\
\hline 2011 CAIST, Lee $e^{23}$ & Cilostazol vs. Aspirin & $100 \%$ & $3 \mathrm{M}$ & $<48 \mathrm{~h}$ & is & 458 & 231 & 227 & $62 \%$ & 63 & $65 \%$ & $35 \%$ \\
\hline 2011 JASAP, Uchiyama $a^{24}$ & A_D vs. Aspirin & $100 \%$ & $15.3 \mathrm{M}$ & & is & 1291 & 652 & 639 & $72 \%$ & 66 & $89 \%$ & $41 \%$ \\
\hline 2011 TOSS2, Kwon ${ }^{25}$ & A_Ci vs. A_C & $100 \%$ & $7 \mathrm{M}$ & & is & 457 & 232 & 225 & $52 \%$ & 65 & $72 \%$ & $43 \%$ \\
\hline 2012 ECLIPse, Han $^{26}$ & A_Ci vs. Aspirin & $100 \%$ & зм & & is & 203 & 100 & 103 & $75 \%$ & 65 & $57 \%$ & $29 \%$ \\
\hline 2012 Nakamuraa & A_Ci vs. Aspirin & $100 \%$ & $6 \mathrm{M}$ & $<48 \mathrm{~h}$ & is & 76 & 38 & 38 & $74 \%$ & 66 & $82 \%$ & $35 \%$ \\
\hline 2013 CHANCE, Wang ${ }^{28}$ & A_C vs. Aspirin & $100 \%$ & зм & $<24 \mathrm{~h}$ & $\mathrm{IS} / \mathrm{TIA}$ & 5170 & 2584 & 2586 & $67 \%$ & 63 & $66 \%$ & $22 \%$ \\
\hline $2014 \mathrm{Y}^{29}$ & A_C vs. Aspirin & $100 \%$ & $1 \mathrm{M}$ & $<48 \mathrm{~h}$ & IS & 574 & 286 & 288 & $55 \%$ & 69 & $73 \%$ & $38 \%$ \\
\hline 2015 CATHARSIS, Uchiyama ${ }^{30}$ & A_Ci vs. Aspirin & $100 \%$ & $24 \mathrm{M}$ & & IS & 163 & 83 & 80 & $66 \%$ & 68 & $77 \%$ & $37 \%$ \\
\hline $2015 \mathrm{He}^{31}$ & A_C vs. Aspirin & $100 \%$ & $2 \mathrm{~W}$ & $<72 \mathrm{~h}$ & IS/TIA & 690 & 343 & 347 & $57 \%$ & 62 & $68 \%$ & $42 \%$ \\
\hline $2015 \mathrm{YY}^{32}$ & A_C vs. Aspirin & $100 \%$ & $6 \mathrm{M}$ & $<48 \mathrm{~h}$ & IS & 979 & 490 & 489 & $56 \%$ & 69 & $71 \%$ & $34 \%$ \\
\hline 2016 COMPRESS, Hong ${ }^{33}$ & A_C vs. Aspirin & $100 \%$ & $1 \mathrm{M}$ & $<48 \mathrm{~h}$ & is & 358 & 178 & 180 & $64 \%$ & 67 & $67 \%$ & $33 \%$ \\
\hline $2016 \operatorname{SOCRATES}(\mathrm{A})$, Wang $^{34}$ & Ticagrelor vs. Aspirin & $100 \%$ & $3 \mathrm{M}$ & $<24 \mathrm{~h}$ & $\mathrm{IS} / \mathrm{TIA}$ & 3858 & 1933 & 1925 & $63 \%$ & 64 & $69 \%$ & $25 \%$ \\
\hline 2017 MAESTRO, Han $^{35}$ & Triflusal vs. Clopidogrel & $100 \%$ & $32 \mathrm{M}$ & & is & 784 & 391 & 393 & $68 \%$ & 61 & $61 \%$ & $29 \%$ \\
\hline 2017 Zuo $0^{36}$ & A_C vs. Aspirin & $100 \%$ & $3 \mathrm{M}$ & & $\mathrm{IS} / \mathrm{TIA}$ & 200 & $66 / 66$ & 68 & $61 \%$ & 61 & $65 \%$ & $32 \%$ \\
\hline 2018 PICASSO, Kim ${ }^{37}$ & Cilostazol vs. Aspirin & $100 \%$ & $23 \mathrm{M}$ & & $\mathrm{IS} / \mathrm{TIA}$ & 1534 & 766 & 768 & $62 \%$ & 65 & $89 \%$ & $33 \%$ \\
\hline 2019 ADS, Aoki ${ }^{38}$ & A_Ci vs. Aspirin & $100 \%$ & $3 M$ & $<48 \mathrm{~h}$ & is & 1201 & 600 & 601 & $66 \%$ & 69 & $76 \%$ & $32 \%$ \\
\hline 2019 PRASTRO-I, Ogawa ${ }^{39}$ & Prasugrel vs. Clopidogrel & $100 \%$ & $25 \mathrm{M}$ & & is & 3747 & 1885 & 1862 & $79 \%$ & 62 & $80 \%$ & $33 \%$ \\
\hline 2019 PRINCE, Wang ${ }^{40}$ & A_Ti vs. A_C & $100 \%$ & $3 \mathrm{M}$ & $<24 h$ & $\mathrm{IS} / \mathrm{TIA}$ & 675 & 336 & 339 & $74 \%$ & 60 & $61 \%$ & $25 \%$ \\
\hline 2020 THALES, Johnston ${ }^{41}$ & A_Ti vs. Aspirin & $42 \%$ & $1 \mathrm{M}$ & $<24 \mathrm{~h}$ & $\mathrm{IS} / \mathrm{T} \mathrm{A}$ & 11016 & 5523 & 5493 & $62 \%$ & 65 & $78 \%$ & $29 \%$ \\
\hline
\end{tabular}

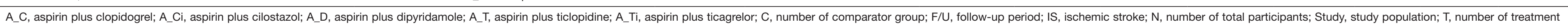
group; TIA, transient ischemic stroke; Tx, time from symptom onset to treatment. 


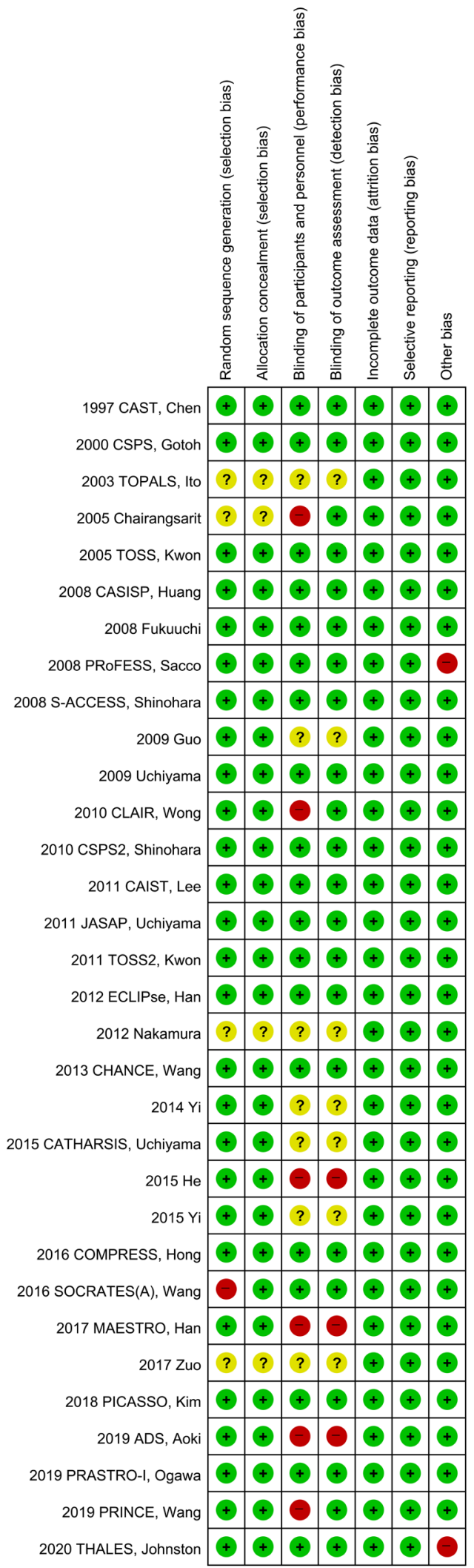

Figure S1 The risk of bias depicted as colors (red: high-risk; green: low-risk; yellow: unclear). 


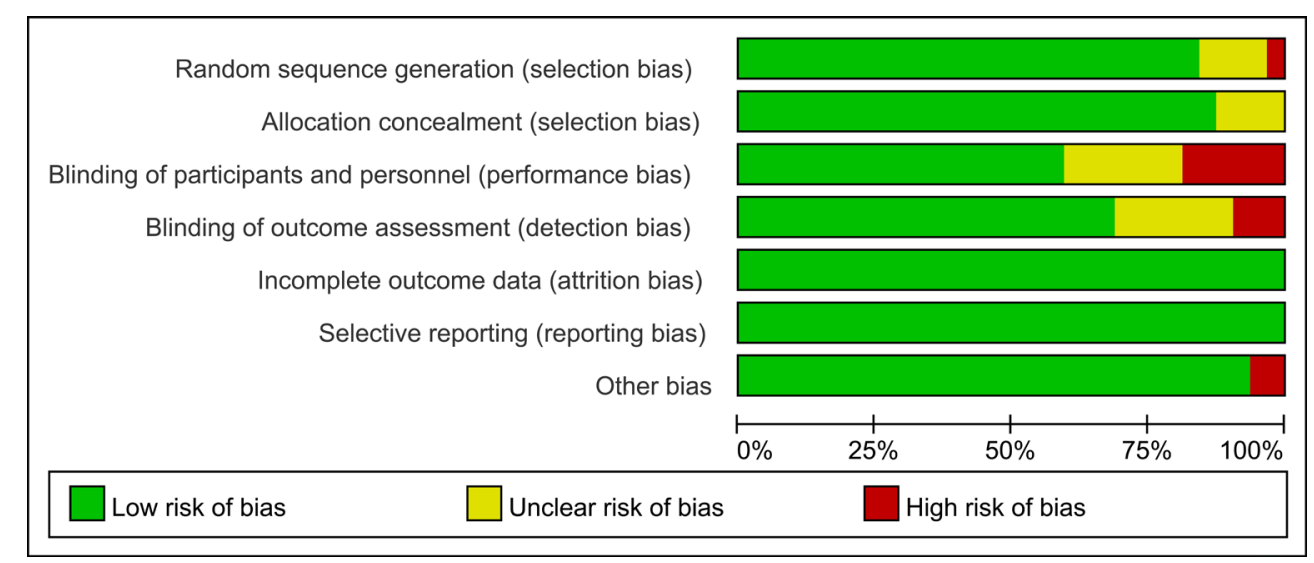

Figure S2 Total graph of the risk of bias of the entire network meta-analysis. Random sequence generation: TOPALS $^{12}$, Chairangsarit ${ }^{13}$, $\mathrm{Nakamura}^{27}$, and Zuo ${ }^{36}$ did not describe their detailed randomization methods; $\mathrm{Wang}^{34}$ is the sub-analysis of the Asian population in the SOCRATES ${ }^{52}$ trial. Blinding of the participants, personnel, and outcome assessment: TOPALS ${ }^{12}$ Guo $^{19}$, Nakamura ${ }^{27}$, Yi2014 ${ }^{29}$, CATHARSIS $^{30}$, Yi2015 ${ }^{32}$, and Zuo ${ }^{36}$ did not describe the methods they used to blind the participants and personnel, or to blind the outcome assessments; $\mathrm{He}^{31}$, MAESTRO $^{35}$, and $\mathrm{ADS}^{38}$ were open-label trials. Other types of bias: PRoFESS ${ }^{17}$ and THALES ${ }^{41}$ were worldwide trials and did not contain only an Asian population. 

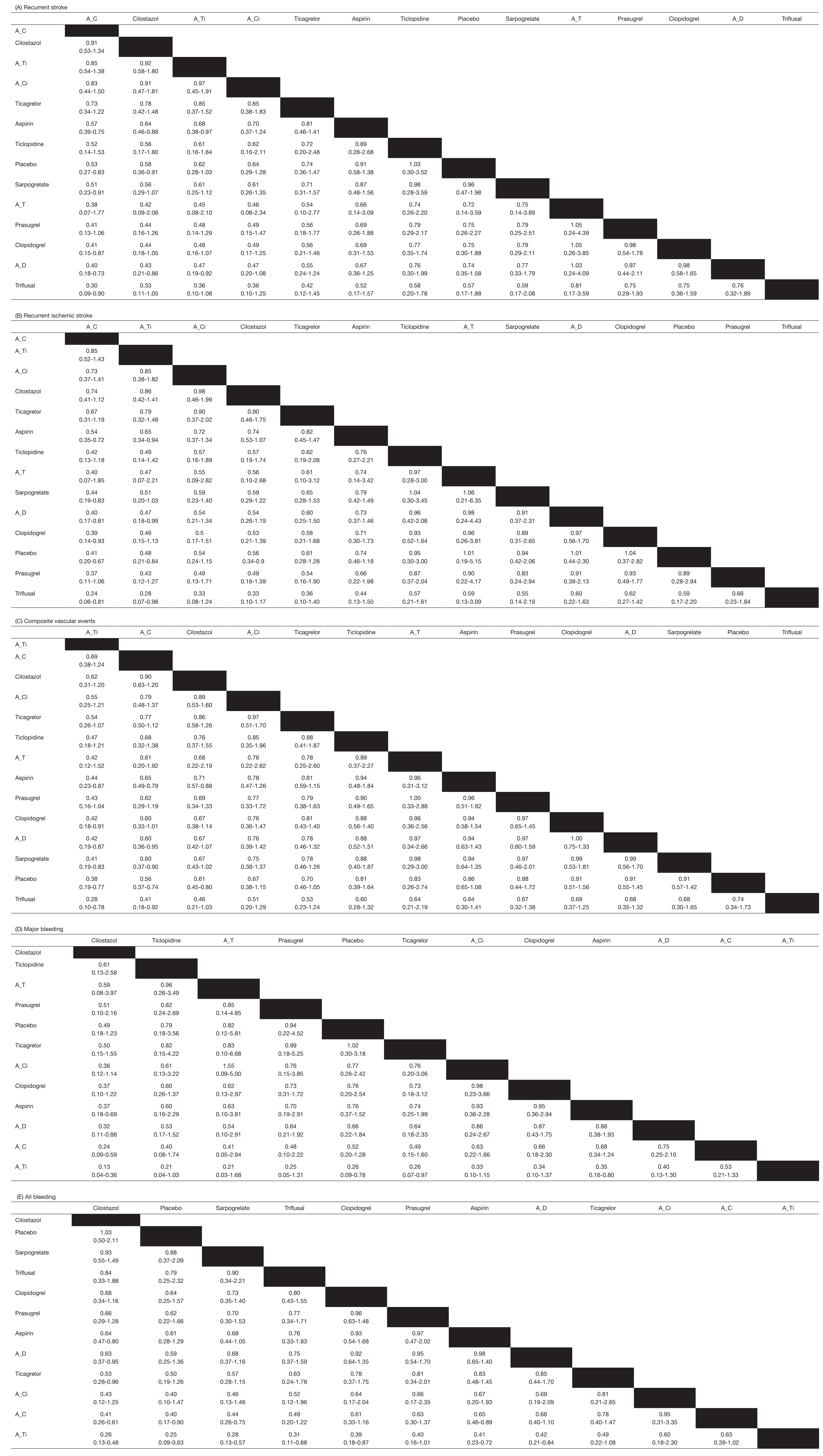
Table S4 Surface under the cumulative ranking curve (SUCRA) values and ranks

\begin{tabular}{|c|c|c|c|c|c|c|c|c|c|c|}
\hline \multirow{2}{*}{ Antiplatelet Regimens } & \multicolumn{2}{|c|}{ Recurrent stroke } & \multicolumn{2}{|c|}{$\begin{array}{c}\text { Recurrent } \\
\text { ischemic stroke }\end{array}$} & \multicolumn{2}{|c|}{ Composite vascular events } & \multicolumn{2}{|c|}{ Major bleeding } & \multicolumn{2}{|c|}{ All bleeding } \\
\hline & SUCRA & Rank & SUCRA & Rank & SUCRA & Rank & SUCRA & Rank & SUCRA & Rank \\
\hline Aspirin & 0.4986558 & 6 & 0.5261077 & 6 & 0.2819797 & 8 & 0.41287955 & 9 & 0.47955682 & 7 \\
\hline Cilostazol & 0.8349288 & 2 & 0.7444500 & 4 & 0.7612962 & 3 & 0.91107045 & 1 & 0.86606136 & 1 \\
\hline Clopidogrel & 0.2695135 & 12 & 0.3274346 & 11 & 0.3518019 & 10 & 0.43967727 & 8 & 0.55560682 & 5 \\
\hline Placebo & 0.4196519 & 8 & 0.3137692 & 12 & 0.2276981 & 13 & 0.61278409 & 5 & 0.81932727 & 2 \\
\hline Prasugrel & 0.2947077 & 11 & 0.2967981 & 13 & 0.4026769 & 9 & 0.61795227 & 4 & 0.50163182 & 6 \\
\hline Sarpogrelate & 0.4045115 & 9 & 0.3679038 & 9 & 0.3468173 & 12 & - & - & 0.80729773 & 3 \\
\hline Ticagrelor & 0.6566538 & 5 & 0.6720212 & 5 & 0.6247096 & 5 & 0.60345227 & 6 & 0.34960455 & 9 \\
\hline Ticlopidine & 0.4582615 & 7 & 0.3974577 & 7 & 0.4912962 & 6 & 0.71462500 & 2 & - & - \\
\hline Triflusal & 0.1497942 & 14 & 0.1176423 & 14 & 0.1153077 & 14 & - & - & 0.70073409 & 4 \\
\hline Aspirin + Cilostazol & 0.7525500 & 4 & 0.7457423 & 3 & 0.6477731 & 4 & 0.46839318 & 7 & 0.25909091 & 10 \\
\hline Aspirin + Clopidogrel & 0.9085288 & 1 & 0.9366154 & 1 & 0.8468385 & 2 & 0.20552500 & 11 & 0.17658409 & 11 \\
\hline Aspirin + Dipyridamole & 0.2407692 & 13 & 0.3317865 & 10 & 0.3479500 & 11 & 0.32975909 & 10 & 0.45227273 & 8 \\
\hline Aspirin + Ticagrelor & 0.7950154 & 3 & 0.8417442 & 2 & 0.9534019 & 1 & 0.03577273 & 12 & 0.03223182 & 12 \\
\hline Aspirin + Ticlopidine & 0.3164577 & 10 & 0.3805269 & 8 & 0.4718558 & 7 & 0.64810909 & 3 & - & - \\
\hline
\end{tabular}


A

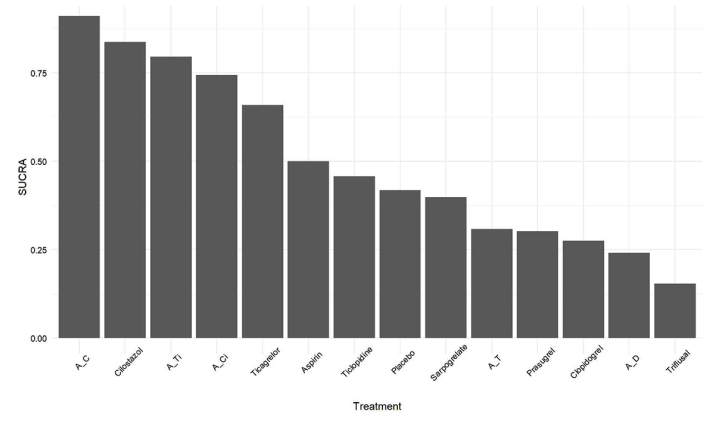

C

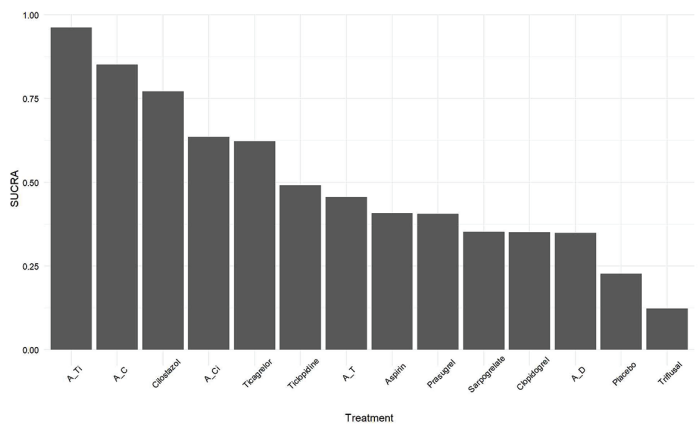

E

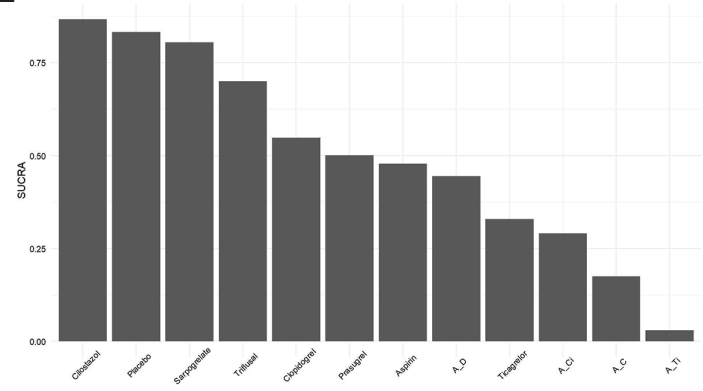

B

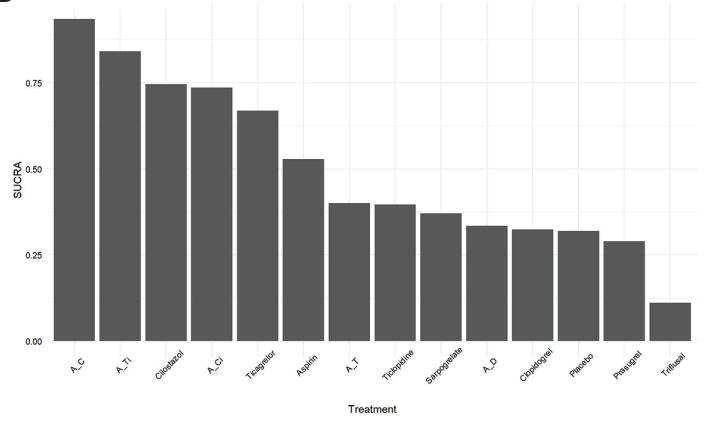

D

Major bleeding

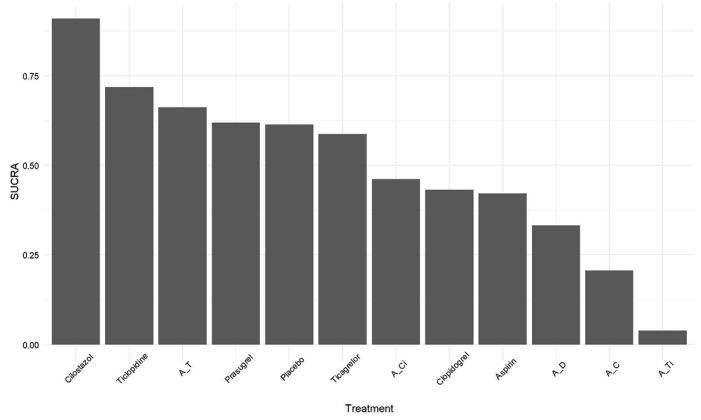

Figure S3 Surface under the cumulative ranking curve (SUCRA) rankograms of the antiplatelet regimens. (A) SUCRA rankogram of the antiplatelet regimens for the recurrent stroke. Aspirin plus clopidogrel ranked first, followed by cilostazol. (B) SUCRA rankogram of the antiplatelet regimens for the recurrent ischemic stroke. Aspirin plus ticagrelor ranked first, aspirin plus clopidogrel second, and cilostazol ranked fourth. (C) SUCRA rankogram of the antiplatelet regimens for the composite vascular events. Aspirin plus ticagrelor ranked first, aspirin plus clopidogrel second, and cilostazol ranked third. (D) SUCRA rankogram of the antiplatelet regimens for the major bleeding. Cilostazol ranked first, and aspirin plus ticagrelor ranked the last. (E) SUCRA rankogram of the antiplatelet regimens for the all bleeding. Cilostazol ranked first, and aspirin plus ticagrelor ranked the last. 
A

Study P-value
A_Ci vs A_C
direct
indirect
network
A_Ti vs A_C
direct
indirect
network
Aspirin vs

Recurrent stroke

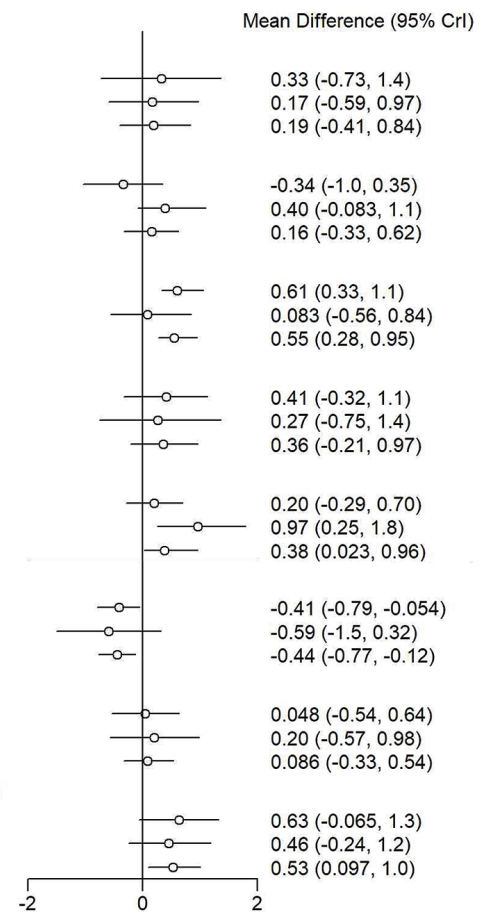

C

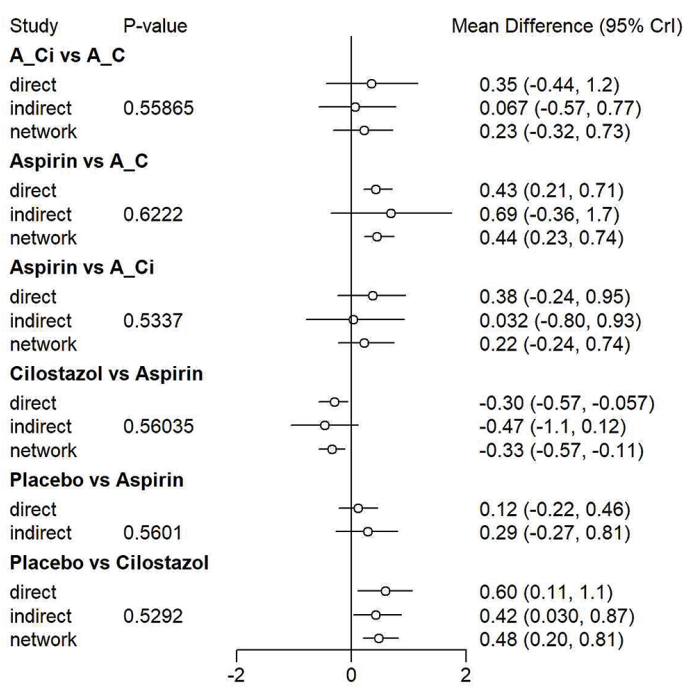

B

Recurrent ischemic stroke

\begin{tabular}{|c|c|}
\hline Study & P-value \\
\hline \multicolumn{2}{|c|}{ A_Civs A_C } \\
\hline $\begin{array}{l}\text { direct } \\
\text { indirect } \\
\text { network }\end{array}$ & 0.7051 \\
\hline \multicolumn{2}{|c|}{ A_Ti vs A_C } \\
\hline $\begin{array}{l}\text { direct } \\
\text { indirect } \\
\text { network }\end{array}$ & 0.04945 \\
\hline \multicolumn{2}{|c|}{ Aspirin vs A_C } \\
\hline $\begin{array}{l}\text { direct } \\
\text { indirect } \\
\text { network }\end{array}$ & 0.14965 \\
\hline \multicolumn{2}{|c|}{ Aspirin vs $\mathrm{A}$ _Ci } \\
\hline $\begin{array}{l}\text { direct } \\
\text { indirect } \\
\text { network }\end{array}$ & 0.69865 \\
\hline \multicolumn{2}{|c|}{ Aspirin vs A_Ti } \\
\hline $\begin{array}{l}\text { direct } \\
\text { indirect } \\
\text { network }\end{array}$ & 0.0464 \\
\hline \multicolumn{2}{|c|}{ Cilostazol vs Aspirin } \\
\hline $\begin{array}{l}\text { direct } \\
\text { indirect } \\
\text { network }\end{array}$ & 0.79355 \\
\hline \multicolumn{2}{|c|}{ Placebo vs Aspirin } \\
\hline $\begin{array}{l}\text { direct } \\
\text { indirect } \\
\text { network }\end{array}$ & 0.7552 \\
\hline \multicolumn{2}{|c|}{ Placebo vs Cilostazol } \\
\hline $\begin{array}{l}\text { direct } \\
\text { indirect } \\
\text { network }\end{array}$ & 0.7608 \\
\hline
\end{tabular}

Mean Difference $(95 \% \mathrm{Crl})$

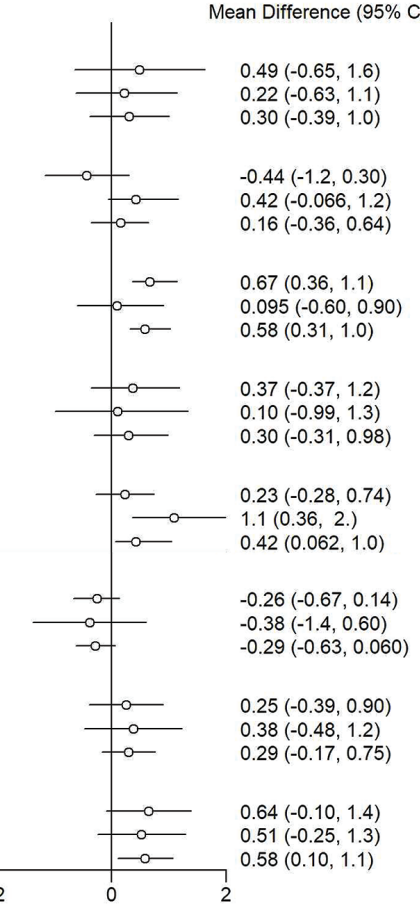

Major bleeding

D

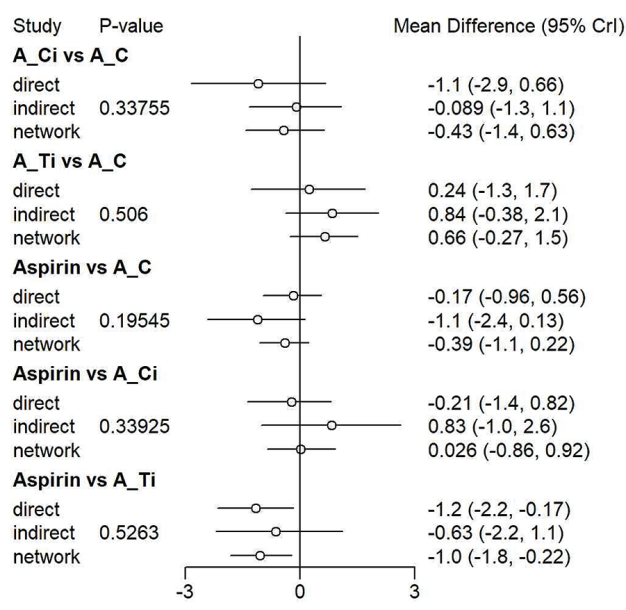

Figure S4 Inconsistency assessments using the node-splitting method. (A) Inconsistency assessments using the node-splitting method for the recurrent stroke. There was no evidence of inconsistencies between the effect estimates of direct and indirect evidence (all P-value of $>0.05$ ). (B) Inconsistency assessments using the node-splitting method for the recurrent ischemic stroke. There was no evidence of inconsistencies between the effect estimates of direct and indirect evidence except in the aspirin $v s$. aspirin plus ticagrelor ( $\mathrm{P}$-value $=0.046$ ). (C) Inconsistency assessments using the node-splitting method for the composite vascular events. There was no evidence of inconsistencies between the effect estimates of direct and indirect evidence (all P-value of $>0.05$ ). (D) Inconsistency assessments using the node-splitting method for the major bleeding. There was no evidence of inconsistencies between the effect estimates of direct and indirect evidence (all P-value of $>0.05$ ). 

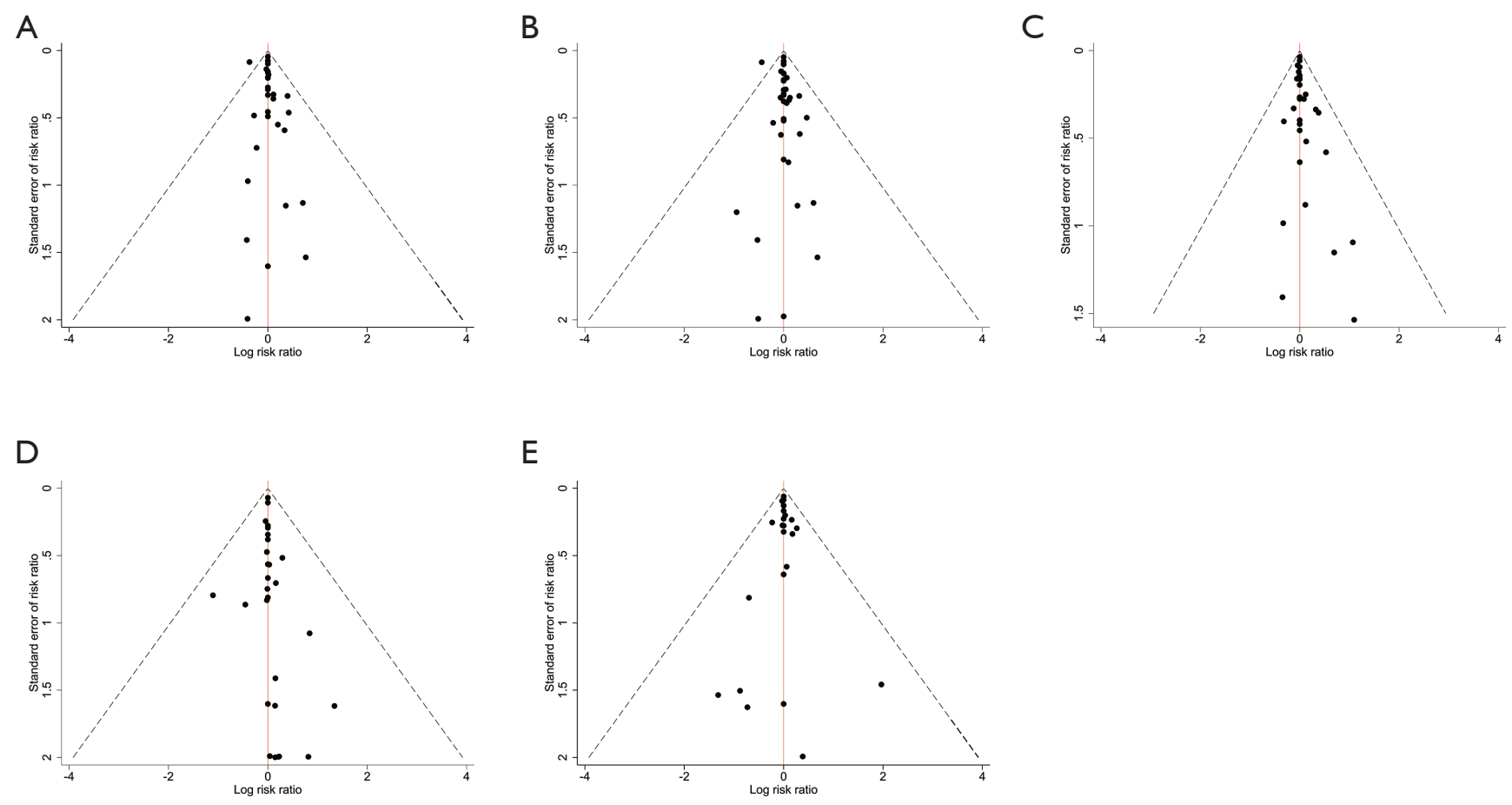

Figure S5 Funnel plots of the antiplatelet regimens of the enrolled trials. The symmetrical shape of the funnel plots demonstrates that there is no evidence of publication bias in this network meta-analysis. (A) Recurrent stroke, (B) Recurrent ischemic stroke, (C) Composite vascular events, (D) Major bleeding, and (E) All bleeding.

A

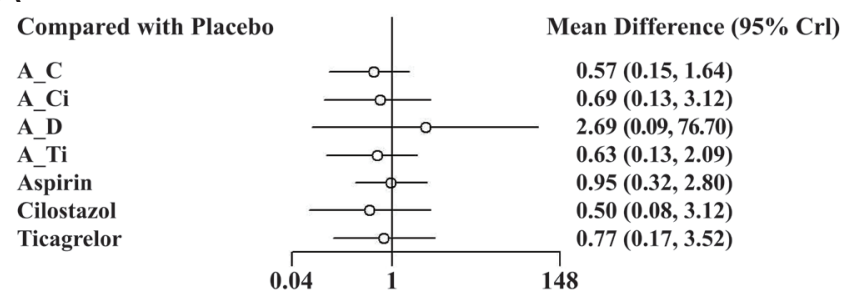

C

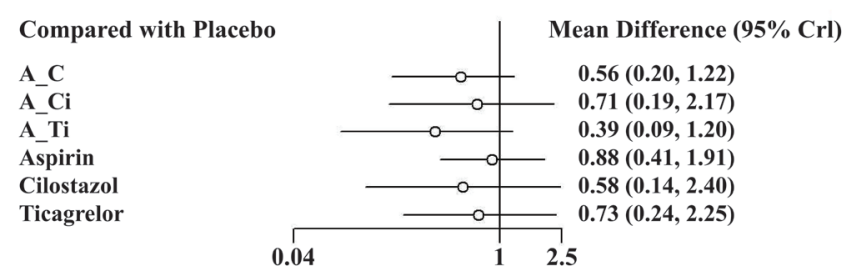

B

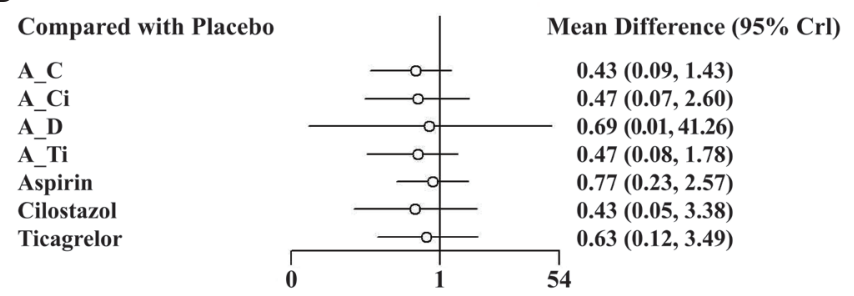

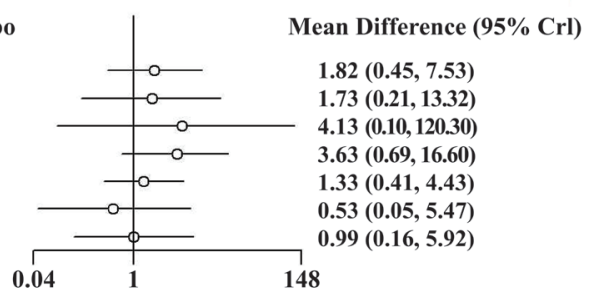

Figure S6 Forrest plots of the antiplatelet regimens compared with placebo for (A) recurrent stroke, (B) recurrent ischemic stroke, (C) composite vascular events, and (D) major bleeding, in the subgroup analysis (less than 72 hours). 
A

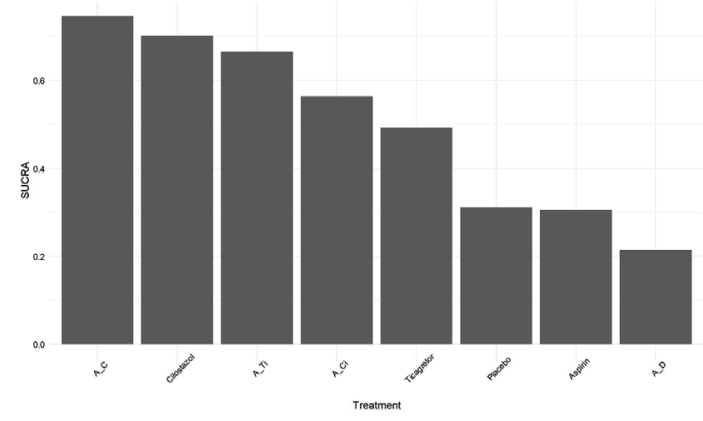

C
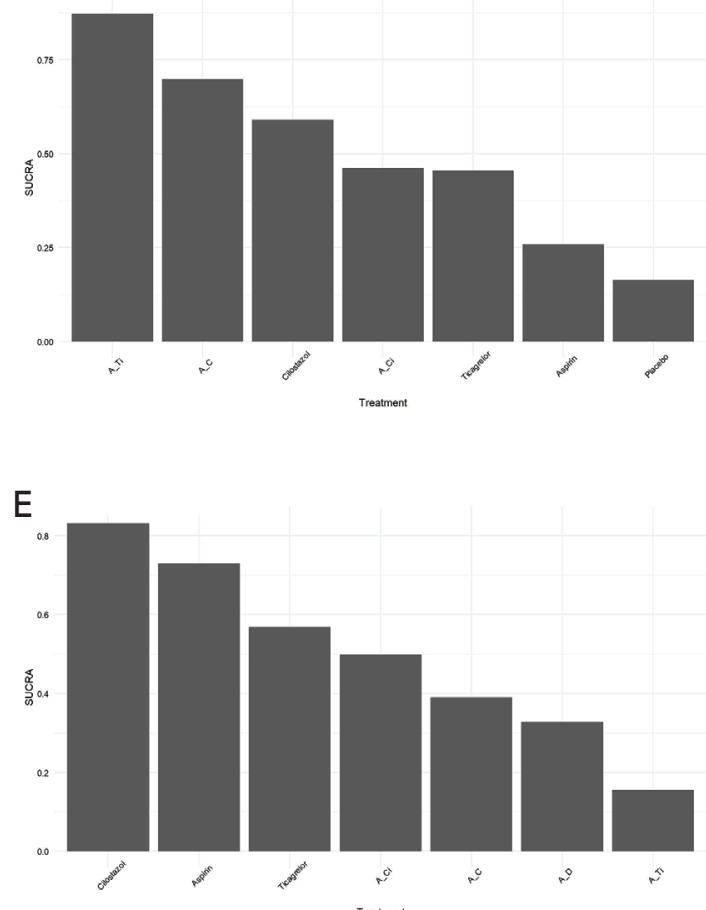

B

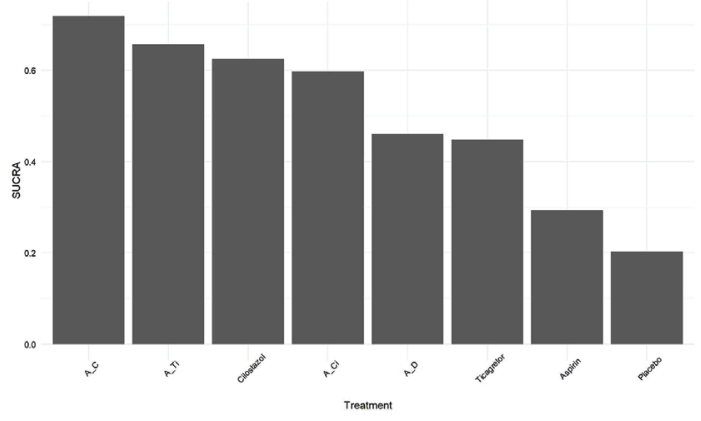

D

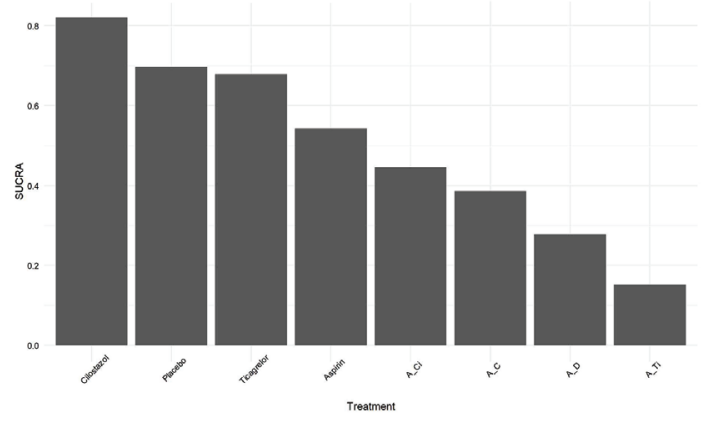

Figure S7 Surface under the cumulative ranking curve (SUCRA) rankograms of the antiplatelet regimens for (A) recurrent stroke, (B) recurrent ischemic stroke, (C) composite vascular events, and (D) major bleeding, in the subgroup analysis (less than 72 hours). 
A

Compared with Placebo

A C

A_Ci

A_D

A_T

Aspirin

Cilostazol

Clopidogrel

Prasugrel

Sarpogrelate

Ticlopidine

Triflusal

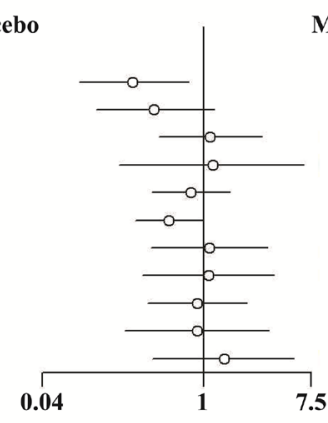

Mean Difference (95\% Crl)

$0.39(0.13,1.22)$

$1.12(0.43,2.97)$

$1.19(0.20,6.48)$

$0.79(0.38,1.63)$

$0.52(0.28,0.99)$

$1.11(0.37,3.32)$

$1.09(0.31,3.74)$

$0.89(0.35,2.25)$

$0.88(0.22,3.38)$

$1.46(0.38,5.36)$
$0.26(0.09,0.75)$

B
Compared with Placebo

A C

A_Ci

A_D

A_T

Aspirin

Cilostazol

Clopidogre

Prasugrel

Sarpogrelate

Ticlopidine

Triflusal



D
Compared with Placebo

A_C

A_Ci

A_D

Aspirin

Cilostazol

Clopidogre

Prasugrel

Ticlopidine

Triflusal
Sarpogrelate

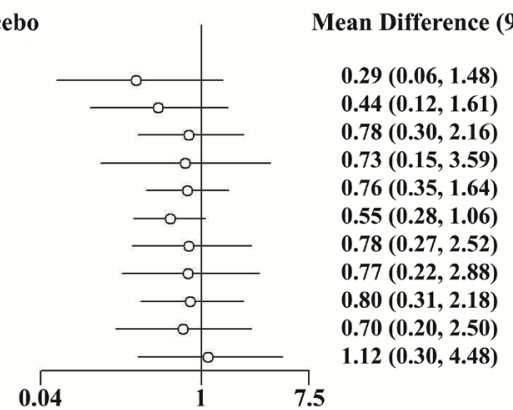

Compared with Placebo

A C

A_Ci

A_D

Aspirin

Cilostazol

Clopidogrel

Prasugrel

Sarpogrelate

Triflusal
Mean Difference (95\% Crl)

$0.23(0.08,0.66)$

$0.44(0.13,1.35)$

$0.91(0.33,2.47)$

$0.88(0.16,4.90)$

$0.66(0.31,1.42)$

$0.52(0.27,1.01)$

$0.93(0.30,2.88)$

$1.00(0.26,3.56)$

$0.83(0.31,2.18)$

$0.86(0.24,3.06)$

$1.49(0.37,5.92)$

Figure S8 Forrest plots of the antiplatelet regimens compared with a placebo for (A) recurrent stroke, (B) recurrent ischemic stroke, (C) composite vascular events, and (D) all bleeding, in the subgroup analysis (not less than 72 hours). 
A

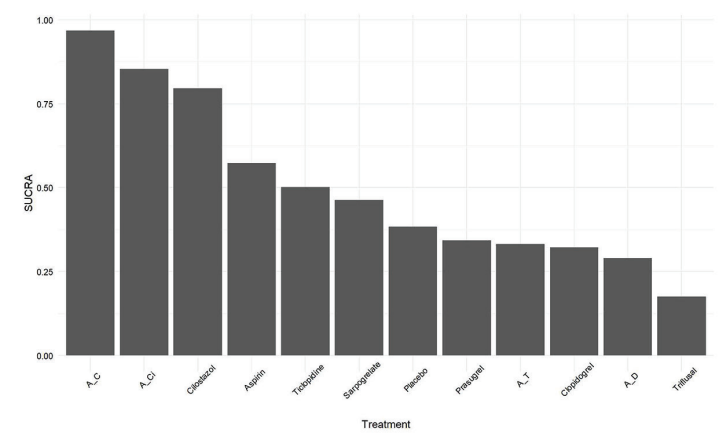

C

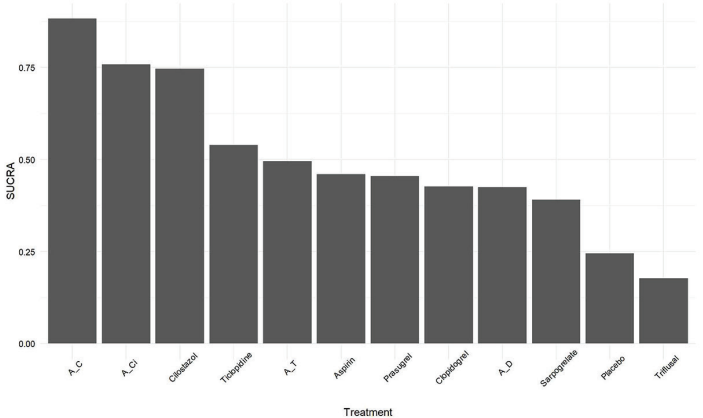

B

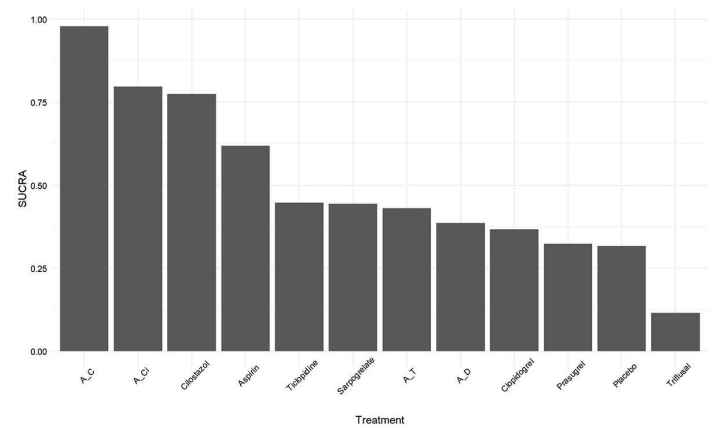

D



Figure S9 Surface under the cumulative ranking curve (SUCRA) rankograms of the antiplatelet regimens for (A) recurrent stroke, (B) recurrent ischemic stroke, (C) composite vascular events, and (D) all bleeding, in the subgroup analysis (not less than 72 hours). 